ESAIM: COCV 21 (2015) 901-923

DOI: $10.1051 / \mathrm{cocv} / 2014049$
ESAIM: Control, Optimisation and Calculus of Variations

www.esaim-cocv.org

\title{
ROBUST OPTIMAL SHAPE DESIGN FOR AN ELLIPTIC PDE WITH UNCERTAINTY IN ITS INPUT DATA
}

\author{
Jesús Martínez-Frutos ${ }^{1}$, Mathieu Kessler $^{2}$ and Francisco Periago ${ }^{2}$
}

\begin{abstract}
We consider a shape optimization problem for an elliptic partial differential equation with uncertainty in its input data. The design variable enters the lower-order term of the state equation and is modeled through the characteristic function of a measurable subset of the spatial domain. As usual, a measure constraint is imposed on the design variable. In order to compute a robust optimal shape, the objective function involves a weighted sum of both the mean and the variance of the compliance. Since the optimization problem is not convex, a full relaxation of it is first obtained. The relaxed problem is then solved numerically by using a gradient-based optimization algorithm. To this end, the adjoint method is used to compute the continuous gradient of the cost function. Since the variance enters the cost function, the underlying adjoint equation is non-local in the probabilistic space. Both the direct and adjoint equations are solved numerically by using a sparse grid stochastic collocation method. Three numerical experiments in 2D illustrate the theoretical results and show the computational issues which arise when uncertainty is quantified through random fields.
\end{abstract}

Mathematics Subject Classification. 35J20, 49J20, 49M20, 65K10.

Received March 10, 2014. Revised July 22, 2014

Published online May 20, 2015.

\section{INTRODUCTION}

Shape optimization amounts to find the optimal shape of a domain which minimizes or maximizes a given criterion (often called 'objective' or 'cost' function). In view of its many possible engineering applications, it is a widely addressed problem and many aspects of shape optimization are addressed in the literature. We refer for instance to $[1,6,9,15,30,39]$ and the references therein.

Probably because of its physical meaning, the compliance is the most studied criterion. Compliance, which is defined as the work done by a load, measures the global rigidity of a physical structure under the action of that load. However, if a shape is optimized for some fixed data (e.g., loads, elastic and/or geometrical material properties, etc..), it is well-known (see for instance [3]) that it could be far from being optimal for small perturbations on that data. Engineering problems are affected by a certain amount of uncertainty in its input data. This uncertainty has several causes (for example, variabilities in the fabrication process of a material)

Keywords and phrases. Robust shape optimization, average approach, stochastic elliptic partial differential equation, relaxation method, Gaussian random fields, elastic membrane.

1 Departamento de Estructuras y Construcción, Universidad Politécnica de Cartagena (UPCT), Campus Muralla del Mar, 30202

Cartagena (Murcia), Spain. jesus.martinez@upct.es

2 Departamento de Matemática Aplicada y Estadística. UPCT. mathieu.kessler@upct.es; f.periago@upct.es 
and should be properly quantified. In many applications, Gaussian fields are the model of choice. Uncertainty Quantification (UQ) is at present a very active field of research in Engineering (see [23]), but is not the object of the present paper.

Several approaches have been suggested to include uncertainty in the shape optimization process [35]. One of them is the robust design optimization (RDO) that aims at obtaining optimal designs less sensitive to variations in the input data [7]. Two methods in this field are the worst-case scenario analysis, which leads to a minmax type optimization problem (see for instance [16,21]), and the so-called average approach [24], where the optimization problem is described in a probabilistic framework. We refer the reader to [7] for an overview of robust optimization. RDO has been applied both to shape and to topology optimization problems e.g. in $[14,21,22,25,37]$.

Unlike the robust optimization, which aims at minimizing the variation of the performance function, other approaches seek to guarantee the system safety in extreme events. To fulfill this new goal, two formulations are commonly used in the literature, namely the reliability-based design optimization and the risk averse optimization. The former is concerned with satisfying a target reliability level by means of failure probabilities $[2,31]$, whereas the latter minimizes a risk functional that quantifies the expected loss related with the damages caused by catastrophic failures $[19,27]$. In this paper we are concerned with the problem of robust shape optimization of elliptic PDEs considering uncertainties in its input data. Thus an average approach which involves the mean and the variance of the compliance in the objective function [24].

It is worth pointing out that most of the engineering-oriented works $[14,25,37]$ dealing with shape optimization under uncertainty are based on mathematical programming models. Thus, when the original problem comes from a continuous model (for example, a partial differential equation like the conductivity equation or the elasticity system), a full discretization of all ingredients entering the problem, namely, the underlying state law (typically by using finite elements), the set of admissible shapes and the objective function, is performed at the very beginning. Optimization then applies to the resulting discrete (finite-dimensional) model.

On the contrary, mathematical-oriented papers typically address continuous (infinite-dimensional) optimization problems. A few recent works have for example addressed the issue of shape optimization under uncertainty by using the average approach, see $[11,18]$. Three common features of these two works are: (1) uncertainty enters in the problem formulation through the loads; (2) only the expected value of the deterministic objective function is considered. Although the expectation gives a first indication of robustness, it is reasonable, in order to achieve a more robust design, to consider as well at least the variance of the objective function. And (3); (approximations of) Gaussian fields as sources of uncertainty in the numerical simulation results presented are not considered. Consequently, the computational complexity involved when Gaussian fields enter into the problem is not shown.

Even if the discrete and continuous methodologies in shape optimization seem at first glance to be equivalent (because even if a continuous model is considered, its numerical resolution necessarily involves a discretization process), they can lead to different results. The subtle difference is that, as proved in some specific optimization problems (see e.g., $[13,28,44]$ ), the diagrams (a) first discretize and then optimize, and (b) first optimize and then discretize are, in general, not equivalent.

In this paper, we consider a shape optimization problem for an elliptic partial differential equation with uncertainty in the diffusion coefficient, the load and the boundary conditions (see Sect. 2.1). The design variable enters the lower order term of the stochastic equation and is subject to a typical measure constraint which makes the problem non-trivial. The objective function is a weighted sum of the expected value of the compliance and of its variance (Sect. 2.2). We focus on the numerical resolution of the problem. For that purpose, since the set of admissible shapes is not convex, we follow the relaxation method. Concretely, the relaxed problem involves a simple convexification of the original set of admissible shapes. The existence of a solution for this new problem and the coincidence of the minimum value of the relaxed cost function with the infimum of the original one is established in Theorem 3.1. The numerical resolution of the relaxed problem is then addressed by following a classical (deterministic) gradient-based minimization algorithm. The novelty here is that, due to the presence of the variance in the cost function, the problem becomes non self-adjoint. Consequently, the explicit computation of 
the continuous gradient (Thm. 4.1) involves the numerical resolution of a Stochastic Elliptic Partial Differential Equation (SEPDE) for the state law and a non-local SEPDE for the adjoint system. We follow a stochastic collocation method, as described in [5], for the numerical resolution of these two problems. To illustrate the theoretical results of this paper, Section 4 describes three numerical experiments where uncertainty appears in the force term (Case 1), in the diffusion coefficient (Case 2), and in the boundary conditions (Case 3). The paper concludes with a final section of further remarks and conclusion where the mathematical and numerical approaches proposed are extended to risk averse shape optimization.

\section{Problem statement}

\subsection{The state law}

Let $D \subset \mathbb{R}^{d}$ be a bounded domain with a Lipschitz boundary $\partial D$, and let $(\Omega, \mathcal{F}, P)$ be a complete probability space. If $X \in L^{1}(\Omega)$ is a real random variable, we denote its expected value by

$$
E(X)=\int_{\Omega} X(\omega) \mathrm{d} P(\omega) .
$$

Consider the stochastic linear elliptic boundary value problem: find a random function $u: \Omega \times \bar{D} \rightarrow \mathbb{R}$ such that $P$-almost surely, the following equation holds

$$
\begin{cases}-\nabla \cdot[a(\cdot, \omega) \nabla u(\cdot, \omega)]+1_{\mathcal{O}} u(\cdot, \omega)=f(\cdot, \omega) & \text { in } D \\ u(\cdot, \omega)=0 & \text { on } \partial D\end{cases}
$$

where $1_{\mathcal{O}}$ stands for the characteristic function associated to a (Lebesgue) measurable subset $\mathcal{O} \subset D$. Here both the gradient $(\nabla)$ and the divergence $(\nabla \cdot)$ operators refer to differentiation with respect to the spatial variable $x \in D$.

In order to rewrite (2.1) in a variational form we consider the following Hilbert spaces:

- $L_{P}^{2}(D \times \Omega)=\left\{f: D \times \Omega \rightarrow \mathbb{R}: \int_{D} E\left[f^{2}\right] \mathrm{d} x<\infty\right\}$.

- $V_{P}=L_{P}^{2}\left(\Omega ; H_{0}^{1}(D)\right)=\left\{v: D \times \Omega \rightarrow \mathbb{R}:\|v\|_{V_{P}}^{2}=\int_{D} E\left[|\nabla v|^{2}\right] \mathrm{d} x<\infty\right\}$.

- $V_{P, a}=\left\{v \in V_{P}:\|v\|_{V_{P, a}}^{2}=\int_{D} E\left[a|\nabla v|^{2}\right] \mathrm{d} x<\infty\right\}$.

Following Babuška et al.[5] we make the following assumptions:

(A1) There exists a random variable $a_{\min }(\omega)$, with $1 / a_{\min } \in L_{P}^{2}(\Omega)$, such that

$$
a(x, \omega) \geq a_{\min }(\omega)>0 \quad \text { a.s. } \omega \in \Omega \text { and a.e. } x \in D .
$$

(A2) $f \in L_{P}^{2}(D \times \Omega)$.

Notice that condition (2.2) is satisfied with a lognormal diffusion coefficient. For instance, if

$$
a(x, \omega)-a_{0}(x)=\exp \left(\sum_{n=1}^{N} b_{n}(x) Y_{n}(\omega)\right), \quad Y_{n} \sim N(0,1) \text { independent and identically distributed (i.i.d.), }
$$

with $a_{0}(x) \geq \alpha_{0}>0$ a.e. $x \in D$.

The variational or weak form of problem (2.1) is:

$$
\text { find } u \in V_{P, a} \text { such that } \int_{D} E\left[a \nabla u \cdot \nabla v+1_{\mathcal{O}} u v\right] \mathrm{d} x=\int_{D} E[f v] \mathrm{d} x \quad \forall v \in V_{P, a} .
$$


As a consequence of assumptions (A1) and (A2) above, the Lax-Milgram lemma ensures the existence and uniqueness of a solution to problem (2.3). Moreover, if $f \in L_{P}^{2}(D \times \Omega)$ and $1 / a_{\min } \in L_{P}^{2}(\Omega)$, then $u \in$ $L_{P}^{2}\left(\Omega ; H_{0}^{1}(D)\right)$ and there exists a constant $C_{P}>0$ such that the moment estimates

$$
\|u(\cdot, \omega)\|_{H_{0}^{1}(D)}^{2} \leq C_{P} \frac{\|f(\cdot, \omega)\|_{L^{2}(D)}}{a_{\min }(\omega)} \quad \text { a.s. }
$$

and

$$
\|u\|_{L_{P}^{2}\left(\Omega ; H_{0}^{1}(D)\right)}^{2} \leq C_{P}\left\|1 / a_{\min }\right\|_{L_{P}^{2}(\Omega)}\|f\|_{L_{P}^{2}(D \times \Omega)}
$$

hold (see [5], Lem. 1.2).

\subsection{The robust optimization problem}

The following shape optimization problem with uncertainty is considered:

$$
(\mathrm{OP})\left\{\begin{array}{l}
\text { Minimize in } 1_{\mathcal{O}}: J\left(1_{\mathcal{O}}\right)=\alpha E\left(\int_{D} f u \mathrm{~d} x\right)+(1-\alpha) \operatorname{Var}\left(\int_{D} f u \mathrm{~d} x\right) \\
\text { subject to } \\
1_{\mathcal{O}} \in \mathcal{U}_{L}
\end{array}\right.
$$

where the set of admissible designs $\mathcal{U}_{L}$ is given by

$$
\mathcal{U}_{L}=\left\{1_{\mathcal{O}} \in L^{\infty}(D ;\{0,1\}):|\mathcal{O}|=L|D|, \quad 0<L<1\right\},
$$

$u=u_{\mathcal{O}}(x, \omega)$ is the associated state, i.e., $u$ solves $(2.3)$,

$$
E\left(\int_{D} f u \mathrm{~d} x\right)=\int_{\Omega} \int_{D} f(x, \omega) u(x, \omega) \mathrm{d} x \mathrm{~d} P(\omega)
$$

is the expected value of the compliance, and

$$
\operatorname{Var}\left(\int_{D} f u \mathrm{~d} x\right)=\int_{\Omega}\left(\int_{D} f u \mathrm{~d} x\right)^{2} \mathrm{~d} P(\omega)-\left(\int_{\Omega} \int_{D} f u \mathrm{~d} x \mathrm{~d} P(\omega)\right)^{2}
$$

is its variance. As usual, $|\cdot|$ stands for the Lebesgue measure and $0 \leq \alpha \leq 1$ is a weighting parameter.

Notice that as a consequence of the estimate (2.5), the cost function in problem (OP) is well-defined.

The problem (OP) presents an average approach, commonly used in the field of robust optimization [24], that combines lower statistical moments of the system performance to find a trade-off between performance and robustness. As indicated in the Introduction, the variance of the compliance is used as a dispersion measure that has the effect of increasing the robustness (minimizing the dispersion) of the optimal design w.r.t. the random perturbations that appear in the state law (2.1).

A physical interpretation of problem (OP) follows: the spatial domain $D \subset \mathbb{R}^{d}$ could model a string $(d=1)$ or a membrane $(d=2)$ which is fixed at the end-points of the string or the boundary of the membrane, and $f$ is a vertical load acting on $D$. In this case, $a=E h$, with $E$ the modulus of elasticity and $h$ the thickness. These input data (also the force $f$ ) are taken to be random, which models a certain amount of uncertainty in the material properties $(E)$, in the geometry $(h)$ and in the loads $(f)$. The random output $u(x, \omega)$ then represents the vertical displacement of the point $x \in D$. In problem (OP) we aim at reinforcing a part of the string or the membrane by setting the stiffness of a sub-domain $\mathcal{O}$ of given measure (length in the case of the string and area for the membrane) to 1 , in order to maximize the global rigidity of the new structure. In this case, the compliance is the opposite of the rigidity, which implies that, the smaller the compliance, the larger the rigidity. In other words, in the optimization problem (OP) we search for the best (i.e. the ones that achieves the minimal compliance) shape and position of the domain $\mathcal{O}$ among all domains which have a fixed measure equal to $L|D|$.

The deterministic version of problem (OP) was considered for the first time in [29] (see also [30], Chap. 7). The results in [29] were extended to the case in which the design variable is not a characteristic function but a measure in [10]. More general nonlinear cost functionals were studied in [42]. 


\section{Existence of A SOlution of the RELAXED PROBlem}

In order to ensure the existence of a solution for the deterministic version of problem (OP), some sufficient conditions on the source term $f$ and on the domain $D$ are introduced in [29]. However, a general result which states the existence of a solution for this problem is unknown. It is thus quite reasonable to think that the same applies for the probabilistic version considered in this paper. However, even if (OP) admits a solution, it is very convenient to introduce a relaxed version for the numerical resolution of (OP). This is the main goal of this section. For this purpose, we consider the new problem

$$
(\mathrm{ROP})\left\{\begin{array}{l}
\text { Minimize in } s: J(s)=\alpha E\left(\int_{D} f u \mathrm{~d} x\right)+(1-\alpha) \operatorname{Var}\left(\int_{D} f u \mathrm{~d} x\right) \\
\text { subject to } \quad s \in \overline{\mathcal{U}}_{L}
\end{array}\right.
$$

where now the set of admissible relaxed designs $\overline{\mathcal{U}}_{L}$ is defined as

$$
\overline{\mathcal{U}}_{L}=\left\{s \in L^{\infty}(D ;[0,1]):\|s\|_{L^{1}(D)}=L|D|, \quad 0<L<1\right\}
$$

and $u=u_{s}(x, \omega)$ is the weak solution of problem

$$
\text { find } u \in V_{P, a} \text { such that } \int_{D} E[a \nabla u \cdot \nabla v+s u v] \mathrm{d} x=\int_{D} E[f v] \mathrm{d} x \quad \forall v \in V_{P, a} .
$$

Notice that (ROP) involves a simple convexification of the space of admissible designs for (OP). From now on, we consider the space $L^{\infty}(D ;[0,1])$ endowed with the weak-^ topology.

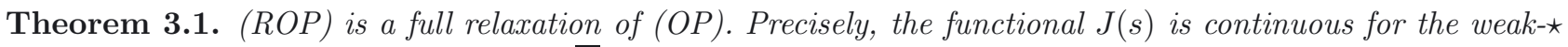
topology. In particular, there exists $s^{\star} \in \overline{\mathcal{U}}_{L}$ such that

$$
\inf _{1_{\mathcal{O}} \in \mathcal{U}_{L}} J\left(1_{\mathcal{O}}\right)=\min _{s \in \overline{\mathcal{U}}_{L}} J(s)=J\left(s^{\star}\right) .
$$

Moreover, if $\alpha=1$, then $J(s)$ is convex.

Proof. Let $s_{n}, s$ be admissible for (ROP) and assume that

$$
s_{n} \rightarrow s \quad \text { weak }-\star \text { in } L^{\infty}(D) .
$$

Denoting by $u_{n}$ and $u$ the corresponding weak solutions of (3.2), we have that the function $v_{n}=u_{n}-u$ solves the problem

$$
\begin{cases}-\nabla \cdot\left[a(\cdot, \omega) \nabla v_{n}(\cdot, \omega)\right]+s_{n} v_{n}(\cdot, \omega)=\left(s-s_{n}\right) u(\cdot, \omega) & \text { in } D \\ v_{n}(\cdot, \omega)=0 & \text { on } \partial D .\end{cases}
$$

From $(2.4)$, it is deduced that $v_{n}(\cdot, \omega)$ is bounded in $H_{0}^{1}(D)$ a.s.. Thus, by the Rellich theorem, there exists a sub-sequence, still denoted by $v_{n}$, and a function $v=v(\cdot, \omega)$ such that

$$
v_{n} \rightarrow v \text { strongly in } L^{2}(D) \text { a.s. }
$$

As a consequence,

$$
u v_{n} \rightarrow u v \quad \text { strongly in } L^{1}(D) \quad \text { a.s. }
$$

and since $s_{n} \rightarrow s \quad$ weak $-\star$ in $L^{\infty}(D)$, we have that

$$
\int_{D}\left(s-s_{n}\right) u v_{n} \mathrm{~d} x \rightarrow 0 \quad \text { a.s. }
$$


By dominated convergence,

$$
\int_{\Omega} \int_{D}\left(s-s_{n}\right) u v_{n} \mathrm{~d} x \mathrm{~d} P(\omega) \rightarrow 0 .
$$

Moreover, from the weak form of (3.3) we have that

$$
\int_{\Omega} \int_{D} a\left|\nabla v_{n}\right|^{2} \mathrm{~d} x \mathrm{~d} P(\omega)+\int_{\Omega} \int_{D} s_{n} v_{n}^{2} \mathrm{~d} x \mathrm{~d} P(\omega)=\int_{\Omega} \int_{D}\left(s-s_{n}\right) u v_{n} \mathrm{~d} x \mathrm{~d} P(\omega) .
$$

Taking limits in this expression, by (3.4), we conclude that

$$
v_{n} \rightarrow 0 \quad \text { in } V_{P, a}
$$

and also strongly in $H_{0}^{1}(D)$ a.s. Since 0 is the single accumulation point of $v_{n}$, the whole sequence converges to 0 . Taking into account (2.7) and (2.8), the continuity of the term of $J(s)$ corresponding to the expected value of the compliance follows easily. The continuity of the variance term is a direct consequence of the result just proved and of the dominated convergence theorem.

The existence of a solution for problem (ROP) is a consequence of the continuity of $J(s)$ and of the compactness of $\overline{\mathcal{U}}_{L}$ w.r.t. the weak- $\star$ topology. By density, the infimum of $J\left(1_{\mathcal{O}}\right)$ in $\mathcal{U}_{L}$ equals the minimum of $J(s)$ in $\overline{\mathcal{U}}_{L}$.

Let us now take $\alpha=1$, i.e., the cost function $J(s)$ only involves the expectation of the compliance. Since $u_{s}$ achieves the minimum of the functional

$$
\frac{1}{2} \int_{\Omega} \int_{D}\left[a|\nabla v|^{2}+s v^{2}\right] \mathrm{d} x \mathrm{~d} P(\omega)-\int_{\Omega} \int_{D} f v \mathrm{~d} x \mathrm{~d} P(\omega),
$$

by $(3.2)$,

$$
\begin{aligned}
-\frac{1}{2} J(s) & =-\frac{1}{2} \int_{\Omega} \int_{D}\left[a|\nabla u|^{2}+s u^{2}\right] \mathrm{d} x \mathrm{~d} P(\omega) \\
& =\min _{v \in V_{P, a}}\left\{\frac{1}{2} \int_{\Omega} \int_{D}\left[a|\nabla v|^{2}+s v^{2}\right] \mathrm{d} x \mathrm{~d} P(\omega)-\int_{\Omega} \int_{D} f v \mathrm{~d} x \mathrm{~d} P(\omega)\right\},
\end{aligned}
$$

which is concave as a minimum of affine functions. This proves that $J(s)$, with $\alpha=1$, is convex.

\section{Numerical Resolution of the Relaxed Problem}

For the numerical resolution of problem (ROP) we use a gradient-based optimization algorithm. Precisely, we shall use the well-known (in structural optimization) Method of Moving Asymptotes (MMA) as described in $[40,41]$. We first focus on the numerical computation of the continuous gradient of the cost function in problem (ROP).

\subsection{Explicit computation of the continuous gradient}

Theorem 4.1. The functional $J(s)$ is Gâteaux differentiable at each admissible $s \in \overline{\mathcal{U}}_{L}$. Moreover, its derivative at $s$ is given by

$$
\begin{aligned}
J^{\prime}(s)(x)= & -\alpha \int_{\Omega} u_{s}^{2}(x, \omega) \mathrm{d} P(\omega) \\
& +(1-\alpha) \int_{\Omega} u_{s}(x, \omega) p_{s}(x, \omega) \mathrm{d} P(\omega) \\
& +2(1-\alpha)\left(\int_{\Omega} \int_{D} f u_{s} \mathrm{~d} x \mathrm{~d} P(\omega)\right) \int_{\Omega} u_{s}^{2}(x, \omega) \mathrm{d} P(\omega)
\end{aligned}
$$


where $u_{s}$ solves the direct problem (3.2) and the adjoint state $p_{s}$ is the solution of the following non-local stochastic elliptic problem: find $p_{s} \in V_{P, a}$ such that

$$
\int_{\Omega} \int_{D}\left[a \nabla p_{s} \cdot \nabla v+s p_{s} v\right] \mathrm{d} x \mathrm{~d} P(\omega)=-2 \int_{\Omega}\left[\int_{D} f u_{s} \mathrm{~d} x \int_{D} f v \mathrm{~d} x\right] \mathrm{d} P(\omega) \quad \forall v \in V_{P, a} .
$$

Proof. Let $\hat{s} \in L^{\infty}(D ;[0,1])$ be an admissible direction, i.e., for $\varepsilon>0$ small enough, $s+\varepsilon \hat{s} \in \overline{\mathcal{U}}_{L}$. We first consider the term (2.7) in $J(s)$ corresponding to the expected value of the compliance. To begin with, we notice that the solution $u_{s+\varepsilon \hat{s}}$ associated to the perturbation $s+\varepsilon \hat{s}$ can be decomposed as $u_{s+\varepsilon \hat{s}}=u_{s}+\hat{u}_{\varepsilon}$, where $\hat{u}_{\varepsilon}$ solves the boundary value problem

$$
\begin{cases}-\nabla \cdot\left(a \nabla \hat{u}_{\varepsilon}\right)+(s+\varepsilon \hat{s}) \hat{u}_{\varepsilon}=-\varepsilon \hat{s} u_{s} & \text { in } D \times \Omega \\ \hat{u}_{\varepsilon}=0 & \text { on } \partial D \times \Omega .\end{cases}
$$

Moreover, thanks to the moment estimate (2.5),

$$
\left\|\hat{u}_{\varepsilon}\right\|_{L_{P}^{2}\left(\Omega ; H_{0}^{1}(D)\right)}^{2} \leq \varepsilon C,
$$

where $C>0$ depends on the $L_{P}^{2}$-norm of $1 / a_{\min }$ and $f$. Subtracting the equations for $s+\varepsilon \hat{s}$ and for $s$, multiplying the result by $\left(u_{s+\varepsilon \hat{s}}+u_{s}\right)$, and finally integrating over $\Omega$ and $D$ we get

$$
\begin{aligned}
\int_{\Omega} \int_{D}\left[a\left|\nabla u_{s+\varepsilon \hat{s}}\right|^{2}+(s+\varepsilon \hat{s}) u_{s+\varepsilon \hat{s}}^{2}\right] \mathrm{d} x \mathrm{~d} P(\omega)-\int_{\Omega} \int_{D}\left[a\left|\nabla u_{s}\right|^{2}+s u_{s}^{2}\right] \mathrm{d} x \mathrm{~d} P(\omega) & \\
& =-\varepsilon \int_{\Omega} \int_{D} \hat{s} u_{s}^{2} \mathrm{~d} x \mathrm{~d} P(\omega)-\varepsilon \int_{\Omega} \int_{D} \hat{s} \hat{u}_{\varepsilon} u_{s} \mathrm{~d} x \mathrm{~d} P(\omega) .
\end{aligned}
$$

Therefore,

$$
\frac{E\left(\int_{D} f u_{s+\varepsilon \hat{s}} \mathrm{~d} x\right)-E\left(\int_{D} f u_{s}\right)}{\varepsilon}=-\int_{\Omega} \int_{D} \hat{s} u_{s}^{2} \mathrm{~d} x \mathrm{~d} P(\omega)-\int_{\Omega} \int_{D} \hat{s} \hat{u}_{\varepsilon} u_{s} \mathrm{~d} x \mathrm{~d} P(\omega) .
$$

Applying (2.5) to $u_{s}$ and considering (4.4), a direct application of Holder's inequality shows that the second term in the right-hand side of this expression vanishes as $\varepsilon \rightarrow 0$. The first term in the right-hand side of (4.1) is then derived.

The third term in (4.1) comes from the second term in (2.8) just as a straightforward application of the chain rule and the result just proved.

Finally, let us compute the second term in the right-hand side of (4.1). Using again the decomposition $u_{s+\varepsilon \hat{s}}=u_{s}+\hat{u}_{\varepsilon}$, we have

$$
\begin{aligned}
\int_{\Omega}\left(\int_{D} f u_{s+\varepsilon \hat{s}} \mathrm{~d} x\right)^{2} \mathrm{~d} P(\omega)-\int_{\Omega}\left(\int_{D} f u_{s} \mathrm{~d} x\right)^{2} \mathrm{~d} P(\omega)= & \int_{\Omega}\left(\int_{D} f \hat{u}_{\varepsilon} \mathrm{d} x\right)^{2} \mathrm{~d} P(\omega) \\
& +2 \int_{\Omega}\left(\int_{D} f u_{s} \mathrm{~d} x \int_{D} f \hat{u}_{\varepsilon} \mathrm{d} x\right) \mathrm{d} P(\omega) .
\end{aligned}
$$

By (2.4), the first term in the right-hand side of this expression behaves as $\varepsilon^{2}$ and hence

$$
\lim _{\varepsilon \rightarrow 0} \frac{\int_{\Omega}\left(\int_{D} f u_{s+\varepsilon \hat{s}} \mathrm{~d} x\right)^{2} \mathrm{~d} P(\omega)-\int_{\Omega}\left(\int_{D} f u_{s} \mathrm{~d} x\right)^{2} \mathrm{~d} P(\omega)}{\varepsilon}=\lim _{\varepsilon \rightarrow 0} \frac{2 \int_{\Omega}\left(\int_{D} f u_{s} \mathrm{~d} x \int_{D} f \hat{u}_{\varepsilon} \mathrm{d} x\right) \mathrm{d} P(\omega)}{\varepsilon} .
$$

On the other hand, putting $v=\hat{u}_{\varepsilon}$ in (4.2) yields

$$
\begin{aligned}
2 \int_{\Omega}\left(\int_{D} f u_{s} \mathrm{~d} x \int_{D} f \hat{u}_{\varepsilon} \mathrm{d} x\right) \mathrm{d} P(\omega) & =-\int_{\Omega} \int_{D}\left[a \nabla p_{s} \cdot \nabla \hat{u}_{\varepsilon}+s p_{s} \hat{u}_{\varepsilon}\right] \mathrm{d} x \mathrm{~d} P(\omega) \\
& =\int_{\Omega} \int_{D} \varepsilon \hat{s} u_{s} p_{s} \mathrm{~d} x \mathrm{~d} P(\omega)+\int_{\Omega} \int_{D} \varepsilon \hat{s} \hat{u}_{\varepsilon} p_{s} \mathrm{~d} x \mathrm{~d} P(\omega),
\end{aligned}
$$


the second equality being a consequence of the variational formulation of (4.3). We now are able to compute the limit in (4.6) and, thanks to the estimate (4.4), obtain the desired result.

From (4.1) it is deduced that the gradient of the cost function depends on the solution of the direct problem (3.2), on the solution of its associated adjoint equation (4.2), and on the corresponding integrals over the probability space. We now describe how to solve numerically these three problems.

\subsection{Numerical resolution of the state and adjoint state equations, and numerical approximation of the related statistical quantities}

Following [5] we make the following assumption, which is not too restrictive since in many problems the source of randomness can be approximated by a finite number of random variables (see, for instance, the case of a truncated Karhunen-Loève expansion [32,33]).

Assumption 4.2 (Finite dimensional noise). The random input data take the form:

$$
a(x, \omega)=a\left(x, Y_{1}(\omega), \cdots, Y_{N}(\omega)\right) \quad \text { and } \quad f(x, \omega)=f\left(x, Y_{1}(\omega), \cdots, Y_{N}(\omega)\right)
$$

where $\left\{Y_{n}\right\}_{n=1}^{N}$ are real-valued random variables with mean value zero and unit variance.

Denote by $\Gamma_{n} \equiv Y_{n}(\Omega)$ the image of $Y_{n}, \Gamma=\prod_{n=1}^{N} \Gamma_{n}$ and assume that $\left[Y_{1}, \cdots, Y_{N}\right]$ have a joint probability density function $\rho: \Gamma \rightarrow \mathbb{R}_{+}$, with $\rho \in L^{\infty}(\Gamma)$.

\subsubsection{The direct problem}

For all $x, \omega \mapsto u(x, \omega)$ is measurable with respect to $\mathcal{F}$. As a consequence of Assumption 4.2, the DoobDynkin's lemma therefore ensures that the solution $u(x, \omega)$ of $(3.2)$ can be represented as

$$
u(x, \omega)=u\left(x, Y_{1}(\omega), \cdots, Y_{N}(\omega)\right) .
$$

The stochastic boundary value problem (3.2) thus becomes the following deterministic Dirichlet boundary value problem for an elliptic PDE with an $N$-dimensional parameter:

$$
\text { find } u \in V_{\rho, a} \text { such that } \int_{\Gamma} \rho\left[(a \nabla u, \nabla v)_{L^{2}(D)}+s(u, v)_{L^{2}(D)}\right] \mathrm{d} y=\int_{\Gamma} \rho(f, v)_{L^{2}(D)} \mathrm{d} y, \quad \forall v \in V_{\rho, a} .
$$

Here $(\cdot, \cdot)_{L^{2}(D)}$ stands for the inner product in $L^{2}(D)$ and $V_{\rho, a}$ is the analogue of $V_{P, a}$ with $(\Omega, \mathcal{F}, P)$ replaced by $\left(\Gamma, \mathcal{B}^{N}, \rho d y\right)$, with $\mathcal{B}^{N}$ the $\sigma$-algebra of the Borel sets in $\mathbb{R}^{N}$. This problem is equivalent to

$$
\int_{D}[a(y) \nabla u \cdot \nabla \phi+s u \phi] \mathrm{d} x=\int_{D} f(y) \phi \mathrm{d} x, \quad \forall \phi \in H_{0}^{1}(D), \quad \rho-a . e . \text { in } \Gamma .
$$

Assuming that both $a$ and $f$ admit a smooth extension on the $\rho d y$-zero sets, (4.8) can be extended a.e. in $\Gamma$ w.r.t. the Lebesgue measure.

To approximate numerically the solution of (4.7), the spaces are considered $V_{p, h}=\mathcal{P}_{p}(\Gamma) \otimes H_{h}(D)$, where:

- $H_{h}(D) \subset H_{0}^{1}(D)$ is a standard finite element approximation of $H_{0}^{1}(D)$; and

- $\mathcal{P}_{p}(\Gamma) \subset L_{\rho}^{2}(\Gamma)$ is the span of tensor product of suitable orthogonal polynomials with degree at most $p=\left(p_{1}, \ldots, p_{N}\right)$, i.e., $\mathcal{P}_{p}(\Gamma)=\bigotimes \mathcal{P}_{p_{n}}\left(\Gamma_{n}\right)$, with $\mathcal{P}_{p_{n}}\left(\Gamma_{n}\right)=\operatorname{span}\left(y_{n}^{m}, m=0, \ldots, p_{n}\right), \quad n=1, \ldots, N$.

The numerical algorithm to solve (4.7) consists of three main steps:

Step 1. Project equation (4.7) onto $H_{h}(D)$ :

$$
\int_{D}\left[a(y) \nabla u_{h} \cdot \nabla \phi_{h}+s u_{h} \phi_{h}\right] \mathrm{d} x=\int_{D} f(y) \phi_{h} \mathrm{~d} x, \forall \phi_{h} \in H_{h}(D) \text {, a.e. } y \in \Gamma \text {. }
$$


Step 2. Collocate the above equation at the zeros $y_{k}$ of the corresponding orthogonal polynomials.

Step 3. Build the discrete solution $u_{h, p} \in \mathcal{P}_{p}(\Gamma) \otimes H_{h}(D)$ by interpolating in $y$ the collocated solutions.

\subsubsection{The adjoint problem}

To solve the adjoint problem (4.2) we follow the same lines as for the direct problem just described. In this case, the main difference w.r.t. the direct problem refers to the non-local term that appears in the right-hand side of (4.2). We next explain how to overcome this difficulty.

First, the continuous equation (4.2) is projected onto the discrete space $H_{h}(D)$. Let us denote by $p_{h}(y)$ the solution of this equation. Second, we collocate the resulting equation at the zeros $y_{k}$ of the orthogonal polynomials of $\mathcal{P}_{p}(\Gamma)$. Then, at each node $y_{k}$ of the probability space, we get

$$
\int_{D}\left[a\left(y_{k}\right) \nabla p_{h} \cdot \nabla \phi_{h}+s p_{h} \phi_{h}\right] \mathrm{d} x=\left(-2 \int_{D} f\left(y_{k}\right) u_{h}\left(y_{k}\right) \mathrm{d} x\right) \int_{D} f\left(y_{k}\right) \phi_{h} \mathrm{~d} x .
$$

Hence,

$$
p_{h}\left(y_{k}\right)=c_{k} u_{h}\left(y_{k}\right), \quad \text { with } c_{k}=-2 \int_{D} f\left(y_{k}\right) u_{h}\left(y_{k}\right) \mathrm{d} x .
$$

\subsubsection{Numerical approximation of integrals over the probability space}

As indicated above, to compute the gradient of the cost function and also to compute the cost function of problem (ROP) itself, it is required to approximate numerically integrals over the space $\Gamma$. More precisely, given a continuous function $g: \Gamma \rightarrow \mathbb{R}$ we use a Gauss quadrature formula approximating $\int_{\Gamma} g(y) \hat{\rho}(y) \mathrm{d} y$. Here $\hat{\rho}$ is an auxiliary probability density function that satisfies

$$
\hat{\rho}\left(y_{1}, \ldots, y_{n}\right)=\prod_{n=1}^{N} \hat{\rho}_{n}\left(y_{n}\right) \quad \text { and } \quad\left\|\frac{\rho}{\hat{\rho}}\right\|_{L^{\infty}(\Gamma)}<\infty .
$$

We refer to [5] for more details. Denoting by $y_{k}=\left(y_{1, k_{1}}, y_{2, k_{2}}, \ldots, y_{N, k_{N}}\right)$ a point in $\Gamma$, we introduce, for $n=1, \ldots, N$, the Lagrange basis $\left\{l_{n, j}\right\}_{j=1}^{p_{n}}$ of the space $\mathcal{P}_{p_{n}}$, i.e.,

$$
l_{n, j} \in \mathcal{P}_{p_{n}}\left(\Gamma_{n}\right), \quad l_{n, j}\left(y_{n, k}\right)=\delta_{j k}, \quad j, k=1, \ldots, p_{n}+1
$$

where $\delta_{j k}$ is the Kronecker symbol. Finally, we set $l_{k}(y)=\prod_{n=1}^{N} l_{n, k_{n}}\left(y_{n}\right)$. With this notation, the Gauss quadrature formula $E_{\hat{\rho}}^{p}[g]$ approximating $\int_{\Gamma} g(y) \hat{\rho}(y) \mathrm{d} y$ is given by

$$
E_{\hat{\rho}}^{p}[g]=\sum_{k=1}^{N} \omega_{k} g\left(y_{k}\right), \quad \omega_{k}=\prod_{n=1}^{N} \omega_{k_{n}}, \quad \omega_{k_{n}} \int_{\Gamma_{n}} l_{k_{n}}^{2}(y) \hat{\rho}(y) \mathrm{d} y .
$$

\section{Numerical RESUlts}

Next, we consider three numerical examples for problem (ROP). These examples have been studied previously from a deterministic point of view in [29]. For comparison reasons, we slightly adapt those examples in order to deal with uncertainties in the force term (Case 1), in the diffusion coefficient (Case 2), and in the boundary conditions (Case 3).

The numerical experiments treated in this section are characterized by the following common features:

- Finite element approximation in the physical domain. A stochastic collocation method is used to numerically solve both the direct and adjoint PDE, as well as to perform the integration required to consider the statistical moments of the compliance. The stochastic collocation method requires the resolution of a set of uncoupled deterministic sub-problems in several nodes in the stochastic domain. Each sub-problem is 
discretized in the physical domain through Finite Elements, using P1 elements on a triangular mesh. The maximum allowed element edge length is fixed to a maximum of $h=0.01$ in all the numerical experiments.

- Uncertainty modeling. Two random fields are used to model uncertainty in the first two experiments. In order to satisfy the ellipticity condition (2.2), a lognormal random field is selected for the diffusion coefficient. Consider

$$
F(x)=\exp (\eta(x)+\xi(x) U(x))
$$

where $\eta(x)$ and $\xi(x)$ are the location and scale parameters of the lognormal distribution, that may depend on $x \in D$. The Gaussian random field $U$ is assumed to have a zero mean, a unit variance and the following isotropic squared exponential correlation function:

$$
C\left(x, x^{\prime}\right)=\exp \left[-\sum_{i=1}^{2} \frac{\left(x_{i}-x_{i}^{\prime}\right)^{2}}{l^{2}}\right]
$$

where $x=\left(x_{1}, x_{2}\right), x^{\prime}=\left(x_{1}^{\prime}, x_{2}^{\prime}\right) \in D$, and $l>0$ is the correlation length. The Gaussian random field $U$ is discretized through the truncated Karhunen-Loève expansion [32,33]:

$$
U(x)=\sum_{n=1}^{N} \sqrt{\lambda_{n}} b_{n}(x) Y_{n}(\omega)
$$

where $\lambda_{n}$ and $b_{n}$ are, respectively, the eigenvalues and eigenvectors of the compact and self-adjoint operator

$$
\psi \mapsto \int_{D} C\left(x, x^{\prime}\right) \psi\left(x^{\prime}\right) \mathrm{d} x^{\prime}, \quad \psi \in L^{2}(D),
$$

and $Y_{n}(\omega)$ is a vector of independent normal random variables with zero mean and unit variance.

In this work, the LU triangular decomposition of the covariance matrix proposed by [20] is used in order to generate the random fields simulations. The decay rate of the eigenvalues is analyzed and the size $N$ of the expansion is fixed in order to capture a $95 \%$ of the energy field. More precisely, we set $N$ to be the smallest positive integer such that

$$
\frac{\sum_{n=1}^{N} \lambda_{n}^{h}}{\operatorname{trace}\left(C_{h}\right)} \geq 0.95
$$

where $\lambda_{n}^{h}$ are the numerical approximation of the eigenvalues considered in (5.3), ordered in non-increasing order, and trace $\left(C_{h}\right)$ is the trace of the discrete (over the spatial discretization of $D$ ) correlation function given in (5.2). The parameter $h$ stands for the size of the mesh in the spatial domain. Uncertainty in the third numerical example is fully characterized by one single normal random variable.

- Collocation approximation in stochastic domain. Since the random variables $Y_{n}$ come from a Karhunen-Loève expansion, they are independent standard Gaussian. Hence, the corresponding collocation nodes $y_{k}$ are the Cartesian products determined by the roots of Hermite polynomials. In order to reduce the computational cost related to the full tensor product rules, an isotropic sparse grid, as proposed in [38], has been applied. Given a 1D quadrature rule $\mathcal{Q}^{k}$ of level $k$, the $M$-dimensional Smolyak isotropic product rule is defined as follows:

$$
\mathcal{A}(k, M)=\sum_{k-M+1 \leq|i| \leq k}(-1)^{k+M-|i|}\left(\begin{array}{c}
k+M \\
k+M-|i|
\end{array}\right)\left(\mathcal{Q}^{i_{1}} \otimes \cdots \otimes \mathcal{Q}^{i_{M}}\right) .
$$


The dimension of the product rule is equal to the size $N$ of the Karhunen-Loève expansion. The nonintrusive characteristic of the stochastic collocation method allows to carry out the parallel computation of the deterministic solver at all of the collocation nodes.

- Optimization algorithm. Our robust optimal shape design problem (OP) aims to find a trade-off between optimality and robustness. Actually, the problem should be formulated as a multi-objective optimization problem encompassing two objective functions: the mean and the variance of the compliance. This optimization problem yields a set of optimal solutions in the Pareto sense [34]. In the present work, the set of Pareto optimal solutions is obtained by a previous scalarization of the multi-objective problem into a single-objective one using a weighted-sum approach. To ensure the same order of magnitude [17], the mean and the variance of the compliance are scaled using the following strategy:

$$
J(s)=\alpha \frac{E\left(\int_{D} f u \mathrm{~d} x\right)}{E^{*}}+(1-\alpha) \frac{\operatorname{Var}\left(\int_{D} f u \mathrm{~d} x\right)}{V^{*}}
$$

where $\alpha \in[0,1]$ is the weight balancing the influence of each objective functional. $E^{*}$ and $V^{*}$ are the mean and the variance of the compliance obtained for the initial design which are used as scale factors. The Method of Moving Asymptotes (MMA), developed by Svanberg in [40,41] is used to solve the optimization problem. The MMA solves a sequence of sub-problems generated from local convex approximations of the objective function and its constraints, based on their values and sensitivities. Using the MMA our problem can be reformulated as follows:

$$
\begin{cases}\text { Minimize in } s=\left(s_{1}, \ldots, s_{n}\right): & J(s)+a_{0} z+c y+\frac{1}{2} d y^{2} \\ \text { subject to } & \text { PDE constraint, } \\ & \frac{\|s\|_{L^{1}(D)}}{L|D|}-1-a z-y \leq 0 \\ & 0 \leq s_{j} \leq 1 j=1, \ldots, n \\ & z \geq 0, y \geq 0,\end{cases}
$$

where $n$ is the number of nodes used to discretize the design variable $s, y \in \mathbb{R}$ and $z \in \mathbb{R}$ are "artificial" variables [41], while $a_{0}, a, c$, and $d$ are given real numbers such that $a_{0}>0, a>0, c>0, d \geq 0$, and $c+d>0$. As indicated above, the PDE constraint in problem (ROP) has been discretized and solved by using finite elements in the physical domain and a stochastic sparse grid in the random domain. Following Svanberg's suggestion [41], the values $a_{0}=1, a=0, c=1000$ and $d=0$ are selected for all the numerical experiments. The maximum number of iterations is set to 300 , and the tolerance of the objective function, constraint, and variables is set to $1 \mathrm{e}-6$. We refer the reader to $[40,41]$ for a more detailed description of the algorithm.

\subsection{Case 1: Uncertainty in the force term}

The computational domain is a two dimensional unit square $D=] 0,1\left[{ }^{2}\right.$. The diffusion coefficient $a=1$ and the force term $f(x, y, \omega)$ is the piece-wise function:

$$
f(x, y, \omega)=\left\{\begin{array}{cc}
20 & 0 \leq x \leq 0.4,0 \leq y \leq 1 \\
0.001 & 0.4<x \leq 0.6,0 \leq y \leq 1 \\
f_{2}(x, y, \omega) & 0.6<x \leq 1,0 \leq y \leq 1
\end{array}\right.
$$

where $f_{2}(x, y, \omega)$ is a lognormal random field obtained through the transformations (5.1)-(5.3) with $\eta=2.9761$ and $\xi=0.1980$. An isotropic correlation length $l=0.3$ is considered. The size of the Karhunen-Loève expansion is $N=10$ and captures the $95 \%$ of the energy field. The level of the $1 \mathrm{D}$ quadrature rules is $k=3$. Using P1 finite elements in space and an isotropic sparse grid collocation approximation with Gauss-Hermite collocation 


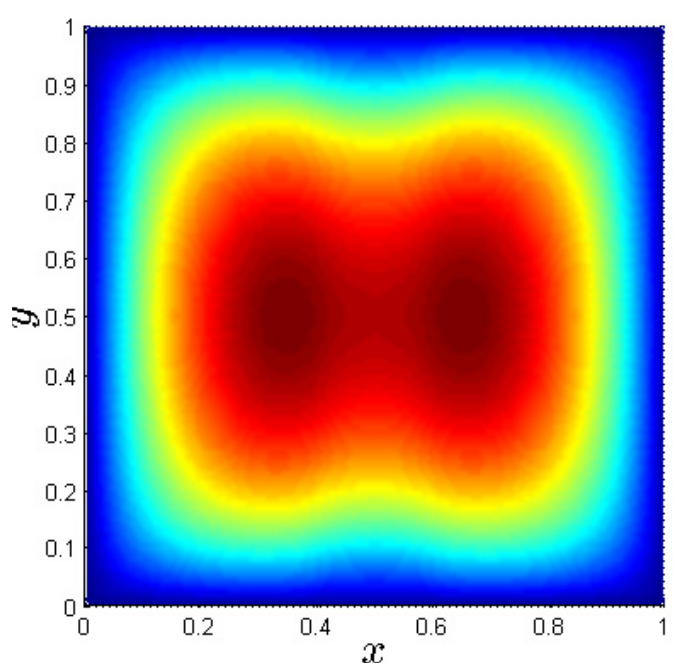

(a)
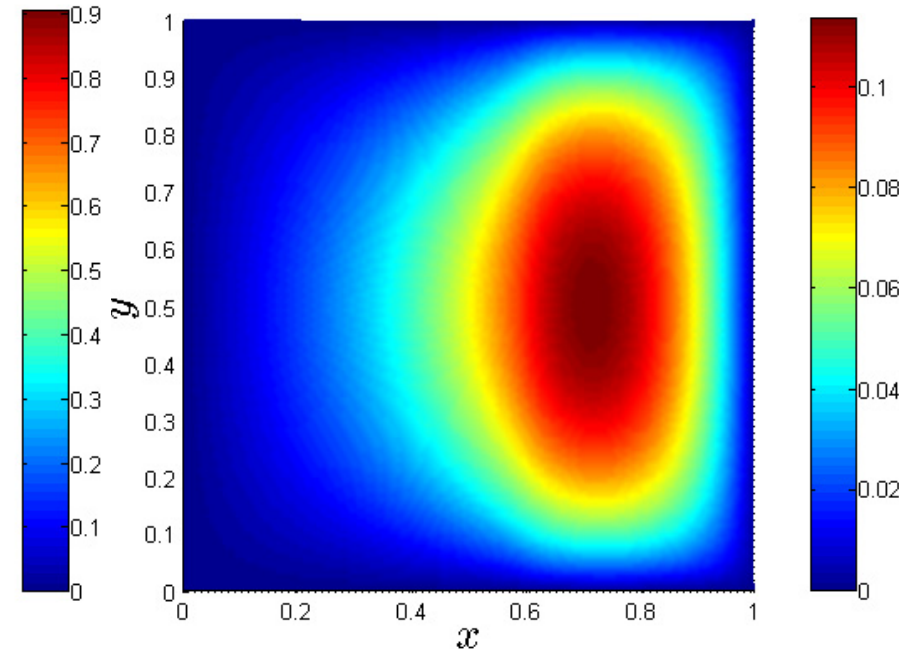

(b)

Figure 1. Case 1. Expectation (a) and standard deviation (b) of $u(x, y, \omega)$ without optimization, i.e. $s=0$.

nodes, the solution $u(x, \omega)$ is computed on each finite element node of 11662 nodes in $D$ and each collocation node of 221 in $\Omega$. The expectation and standard deviation of the uncontrolled (i.e., $s=0)$ solution $u(x, \omega)$ are represented in Figure 1. Due to the uncertainty introduced in the force term, the region $\{0.6 \leq x \leq 1\}$ presents the largest standard deviation.

The optimization problem (ROP) has been solved for values of the weight factor $\alpha=0,0.2,0.4,0.6,0.8,0.85$, $0.9,0.95$ and 1 . The measure constraint is $|\mathcal{O}|=0.1|D|$. In order to analyze the effectiveness of the analytical expression for the sensitivities of the statistical moments, the convergence history of the optimization algorithm, for $\alpha=0$, is plotted in Figure 2. Notice that the objective function presents a peak after the first iteration due to the in-feasibility of the chosen initial design. Once the algorithm has achieved a feasible design, the objective function exponentially decreases until convergence (iteration 53). Figures 3 and 4 show the Pareto front of optimal solutions and the optimal designs $s(x, y)$, respectively. The mean value and variance of the solution $u(x, y, \omega)$ associated with the deterministic optimal solution (which is plotted in Fig. 4f) have been computed and plotted along the Pareto front of optimal solutions (see star in Fig. 3).

The optimal solutions for the robust shape optimization problem can be compared to the deterministic one. In this example, the optimal solution for the case of minimal expectation is very close to the deterministic solution (Figs. 4e-4f). As expected, for the most robust design (minimal variance) all the reinforcement is devoted to strengthen the region of maximum variability of the solution (see Figs. 1b and 4a). When only the expectation of the compliance is considered, the optimal design (Fig. 4e) adopts the region where the displacement is the largest (Fig. 1a). The solutions of the optimization problems for intermediate values of the weight parameter $\alpha=0.2,0.4,0.6,0.8,0.85,0.9$ and 0.95 yield a trade-off between robustness and performance (Fig. 4 ), providing a designer with a set of compromise solutions.

\subsection{Case 2: Uncertainty in the diffusion coefficient}

As in Case 1, the physical domain is the unit square $D=] 0,1\left[{ }^{2}\right.$. In order to produce more realistic results, the lower-order term $s(x, y) u(x, y, \omega)$ in $(3.2)$ has been replaced by $b s(x, y) u(x, y, \omega)$, with $b=100$. The force term is $f(x, y)=100 \sin (3 \pi x) \sin (5 \pi y)$, and the diffusion coefficient $a(x, y, \omega)$ is a lognormal random field obtained 


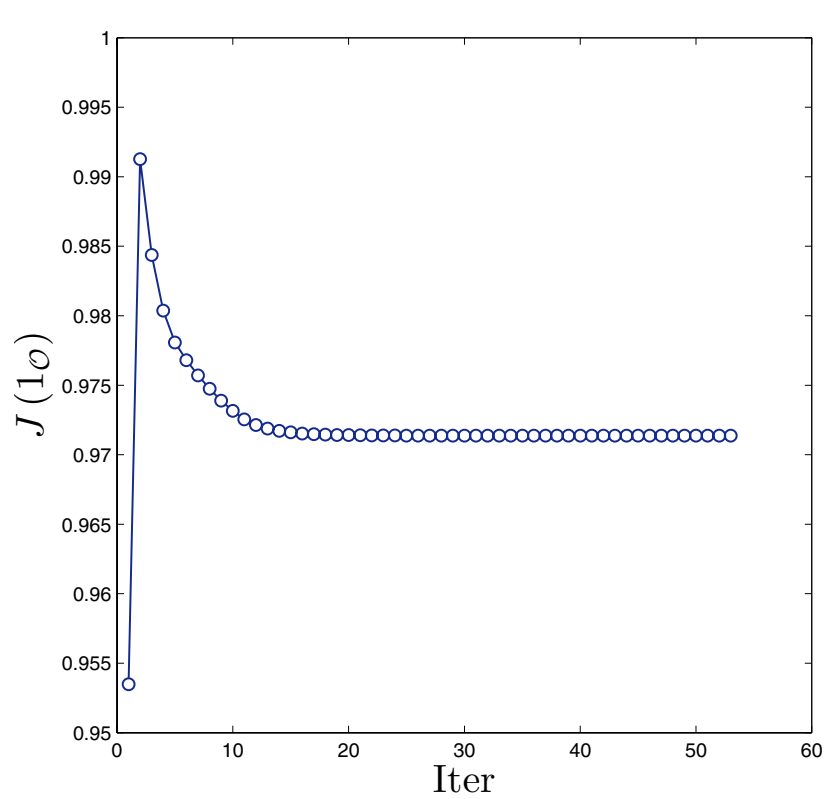

(a)

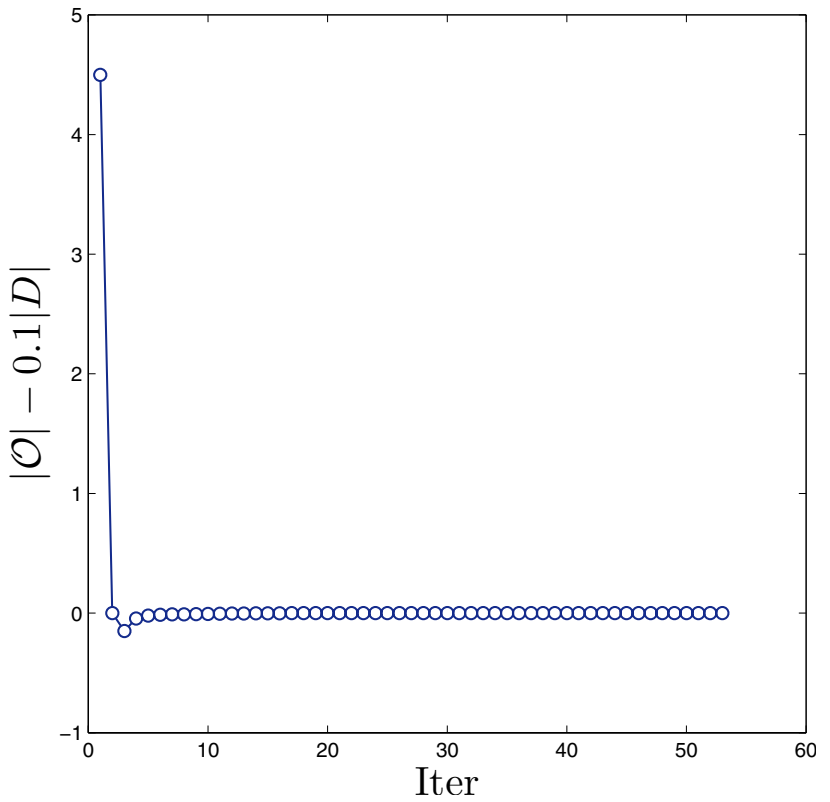

(b)

Figure 2. Case 1. Convergence history of the optimization algorithm for $\alpha=0$. Objective function (a) and measure constraint (b).

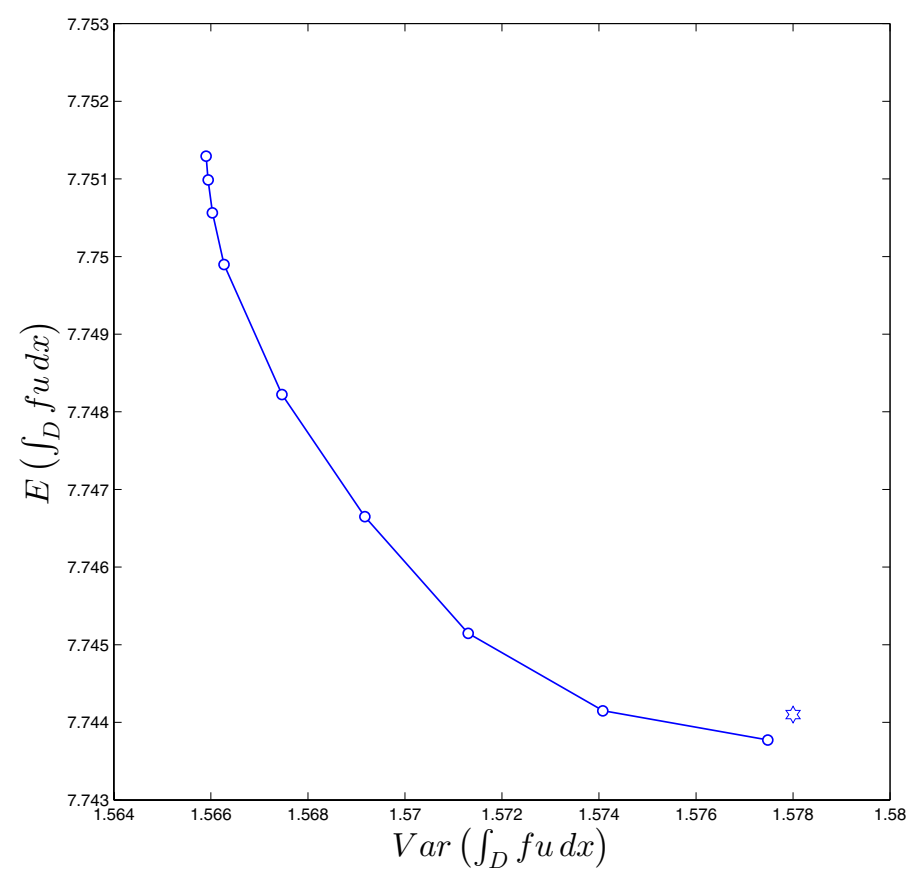

FIgURE 3. Case 1. Pareto front of optimal solutions (circles) and deterministic optimal solution (star). From left to right, and top to bottom, the circles correspond to the values $\alpha=0,0.2$, $0.4,0.6,0.8,0.85,0.9,0.95$ and 1. 


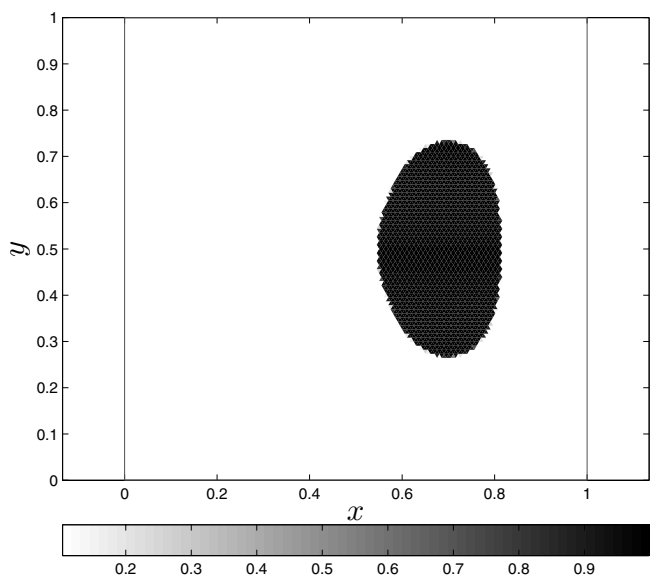

(a) $\alpha=0$

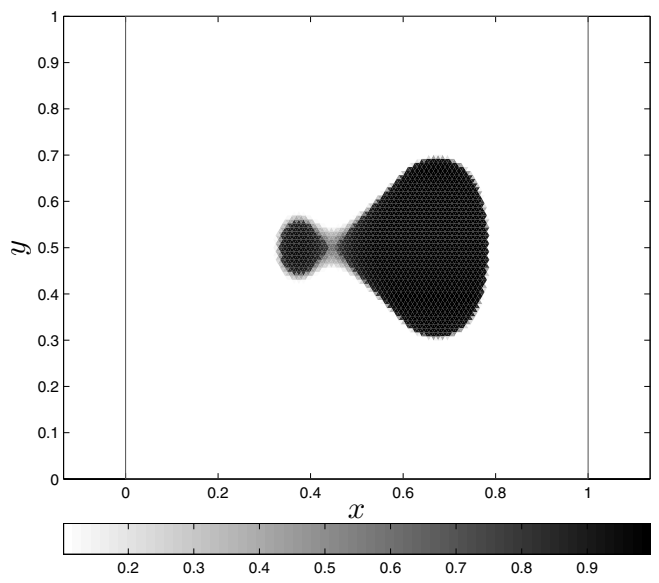

(c) $\alpha=0.85$

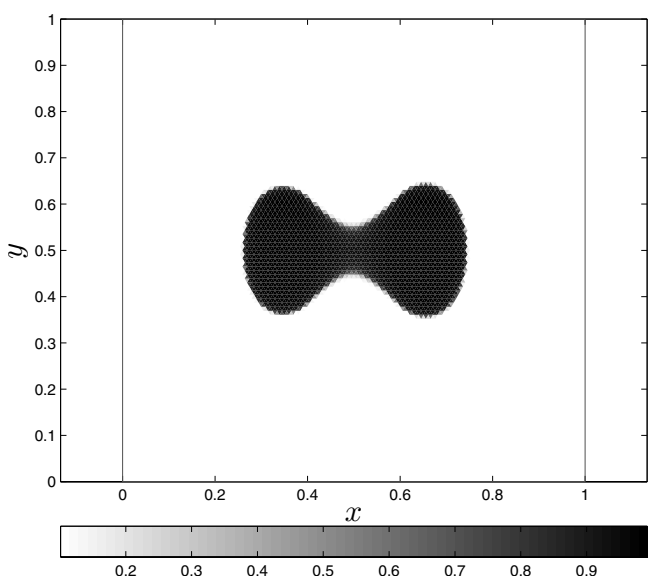

(e) $\alpha=1$

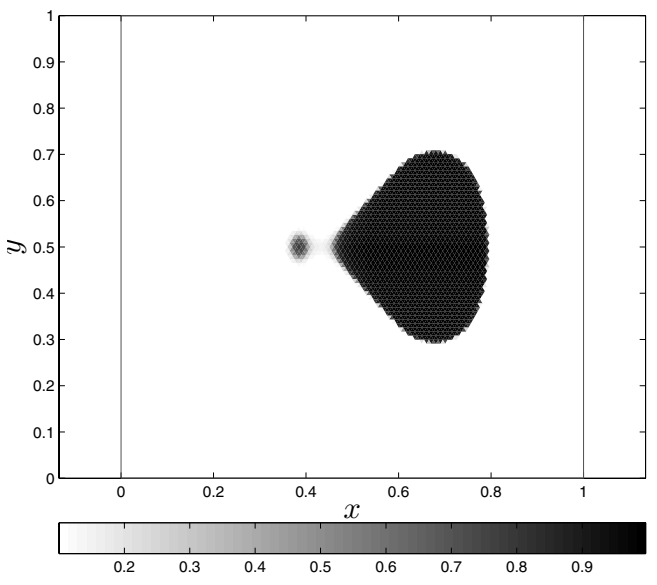

(b) $\alpha=0.8$

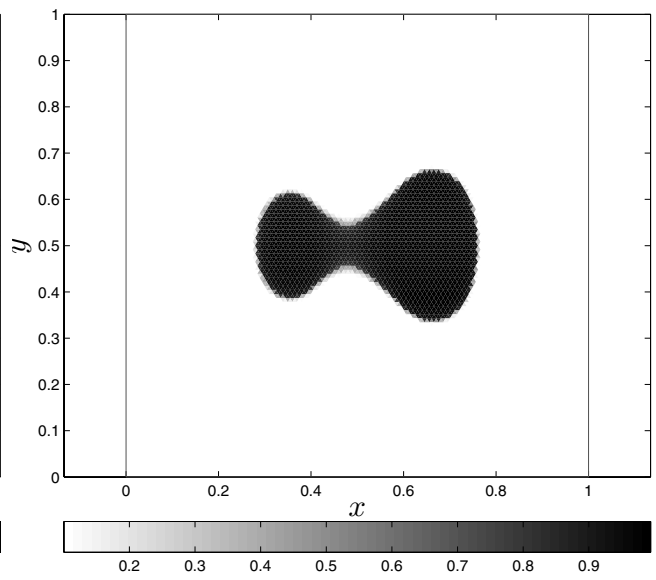

(d) $\alpha=0.95$

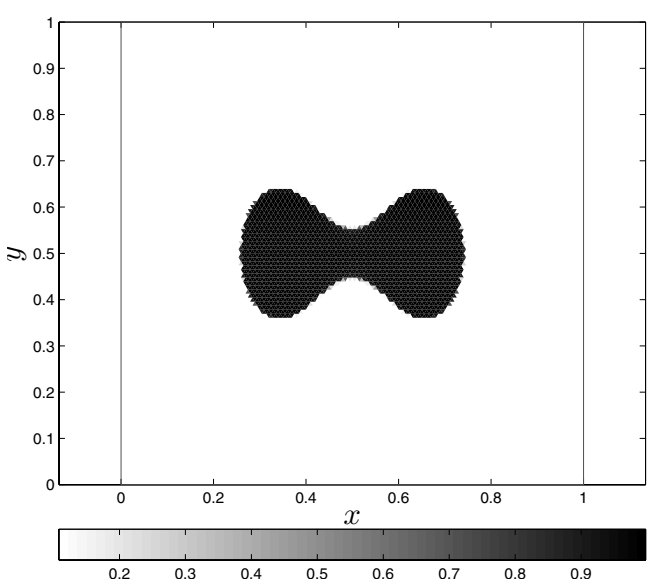

(f) deterministic optimal design

Figure 4. Case 1. Optimal design $s(x, y)$ for different values of $\alpha$. Case (a) corresponds to minimal variance, case (e) minimal expectation, and case (f) optimal deterministic design. 


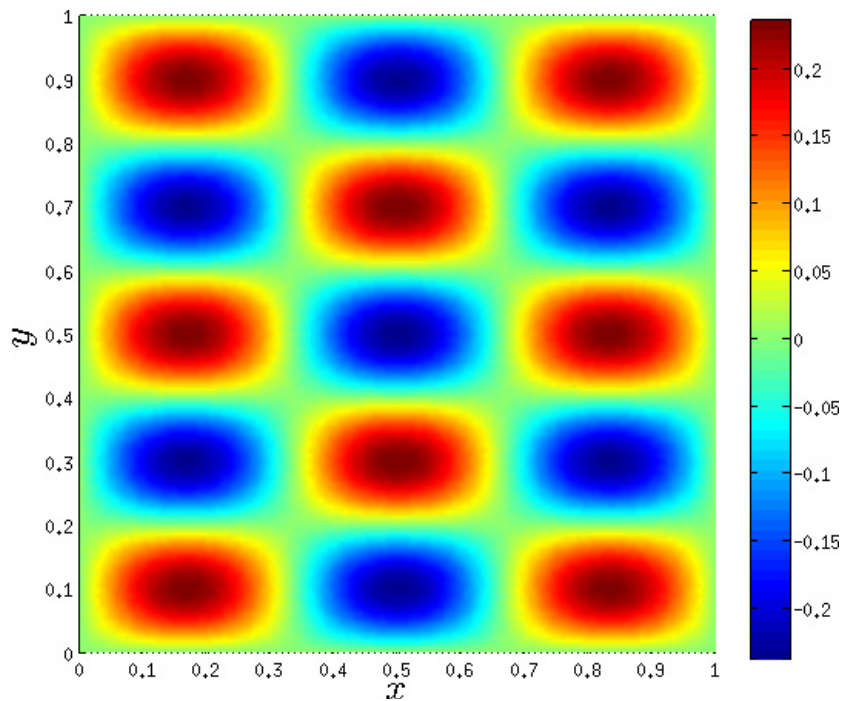

(a)

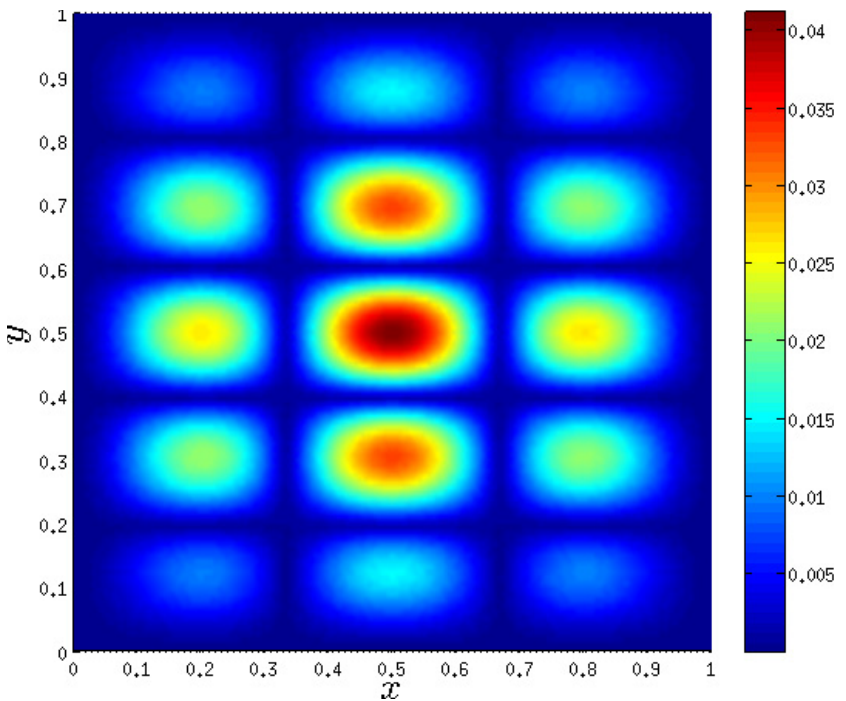

(b)

Figure 5. Case 2. Expectation (a) and standard deviation (b) of $u(x, y, \omega)$ without optimization $(s=0)$.

through the transformations (5.1)-(5.3) with

$$
\begin{aligned}
\eta(x, y) & =\log \left(\frac{1}{(1+\Psi(x, y))^{1 / 2}}\right), \\
\xi(x, y) & =(\log (1+\Psi(x, y)))^{1 / 2}, \\
\Psi(x, y) & =\frac{256}{25}\left(-x^{2}+x\right)^{2}\left(-y^{2}+y\right)^{2} .
\end{aligned}
$$

An isotropic correlation length $l=0.3$ is considered. The size of the Karhunen-Loève expansion is $N=14$ capturing the $95 \%$ of the energy field. The level of the 1D quadrature rules is $k=4$.

Figure 5 plots the expectation and standard deviation of the uncontrolled $(s=0)$ state variable $u(x, \omega)$. The parameter for the measure constraint $L=0.1$. Seven single-objective optimization problems are derived from the multi-objective scalarization by modifying the weight factor $\alpha=0,0.2,0.4,0.6,0.8,0.9,1$. The convergence of the objective function and the evolution of the constraint fulfillment is similar to Case 1 . The solution of the single-objective problems yield different optimal relaxed shapes, which are shown in Figure 7 over the physical domain and in Figure 6 over the objective function domain. Similarly to Case 1, as shown in Figure 7, the minimal expectation design is very close to the deterministic one. However, the design that provides more robustness (minimal variance) consists of a reinforcement in the region of the domain with the largest variability in the diffusion coefficient.

\subsection{Case 3: Uncertainty in the boundary conditions}

The goal of this numerical experiment is to obtain a reinforcement configuration of an elastic membrane less sensitive to modifications in the boundary conditions and with a better performance. Concretely, we consider 


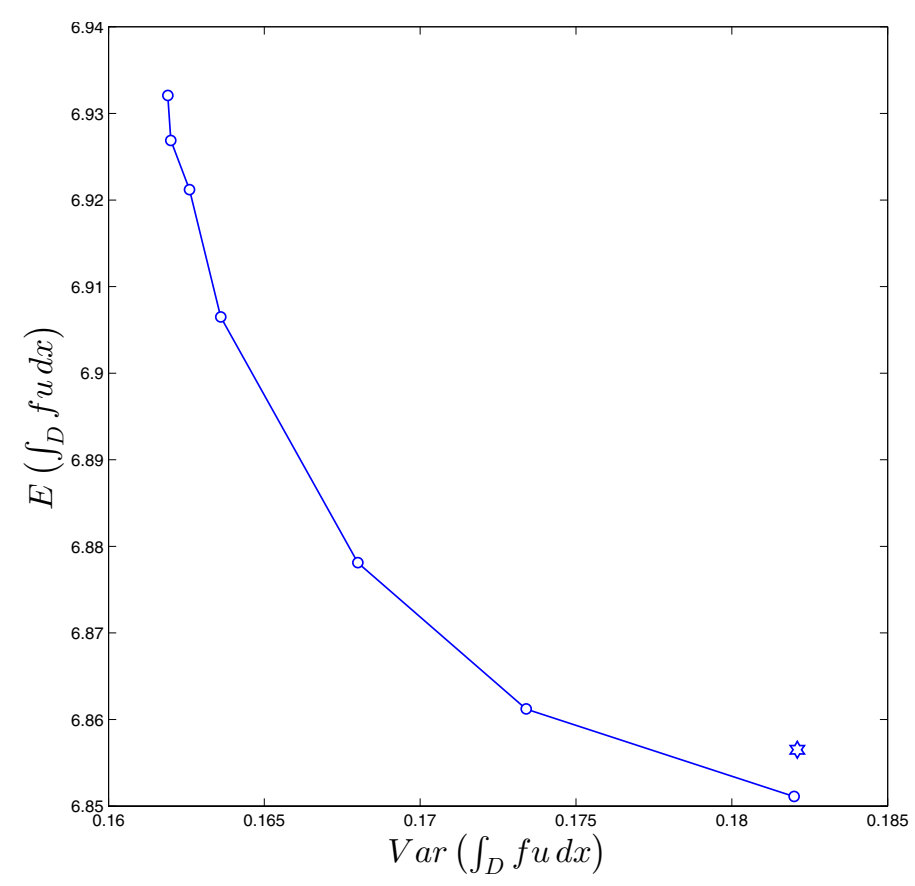

FiguRe 6. Case 2: Pareto front of optimal solutions (circles) and deterministic optimal solution (star). From left to right, and top to bottom, the circles correspond to the values $\alpha=0,0.2$, $0.4,0.6,0.8,0.9$ and 1.

the following boundary value problem with uncertainty in the boundary conditions:

$$
\left\{\begin{aligned}
-\nabla \cdot \nabla u(\cdot, \omega)+s(\cdot) u(\cdot, \omega) & =1 \text { in } D \\
u(\cdot, \omega) & =0 \text { on } \Gamma_{D}(\omega) \\
\frac{\partial u}{\partial \boldsymbol{n}}(\cdot, \omega) & =0 \text { on } \Gamma_{N}(\omega)
\end{aligned}\right.
$$

where the physical domain is the unit circle $D=\left\{(x, y) \in \mathbb{R}^{2}: x^{2}+y^{2}<1\right\}$, and $\boldsymbol{n}$ denotes the unit outward normal vector. The boundary of $D$ is decomposed into two disjoint random parts $\Gamma=\Gamma_{D}(\omega) \cup \Gamma_{N}(\omega)$, with $\Gamma_{D}(\omega) \cap \Gamma_{N}(\omega)=\emptyset . \Gamma_{D}(\omega)$ is parametrized as

$$
\gamma_{D}(\omega):\left[\frac{\omega}{2}, \frac{\pi}{2}-\frac{\omega}{2}\right] \bigcup\left[\frac{\pi}{2}+\frac{\omega}{2}, \pi-\frac{\omega}{2}\right] \bigcup\left[\pi+\frac{\omega}{2}, \frac{3 \pi}{2}-\frac{\omega}{2}\right] \bigcup\left[\frac{3 \pi}{2}+\frac{\omega}{2}, 2 \pi-\frac{\omega}{2}\right] \longrightarrow \mathbb{R}^{2}
$$

where $\omega$ is a random variable that follows a truncated normal distribution with zero mean and $\sigma=\pi / 12 \mathrm{rad}$ standard deviation. The lower/upper truncation point of the distribution is $-2 \sigma /+2 \sigma$. The variational form of problem (5.10) is: find $u \in L_{P}^{2}\left(\Omega ; H^{1}(D)\right)$ such that

$$
\int_{D} E[\nabla u \cdot \nabla v+s u v] \mathrm{d} x=\int_{D} E[f v] \mathrm{d} x \quad \forall v \in L_{P}^{2}\left(\Omega ; H^{1}(D)\right) .
$$




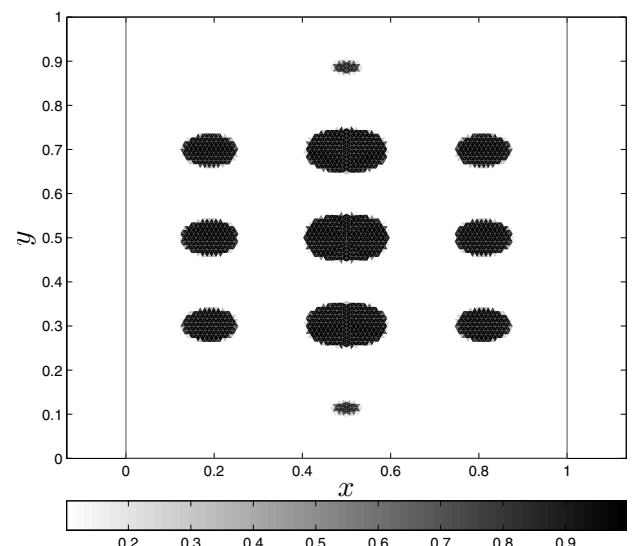

(a) $\alpha=0$

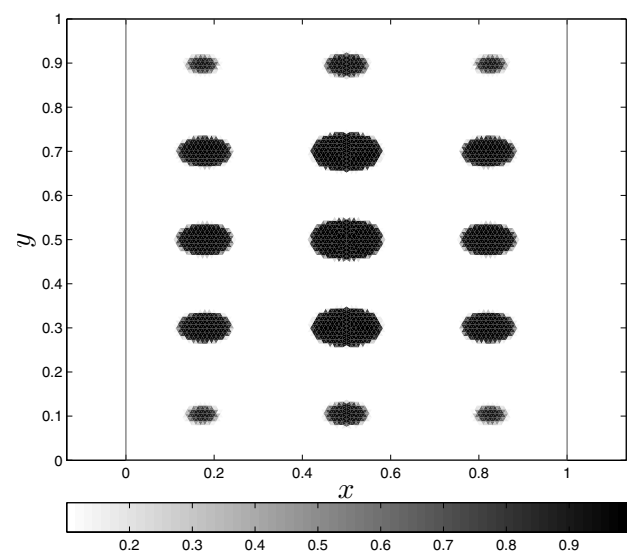

(c) $\alpha=0.8$

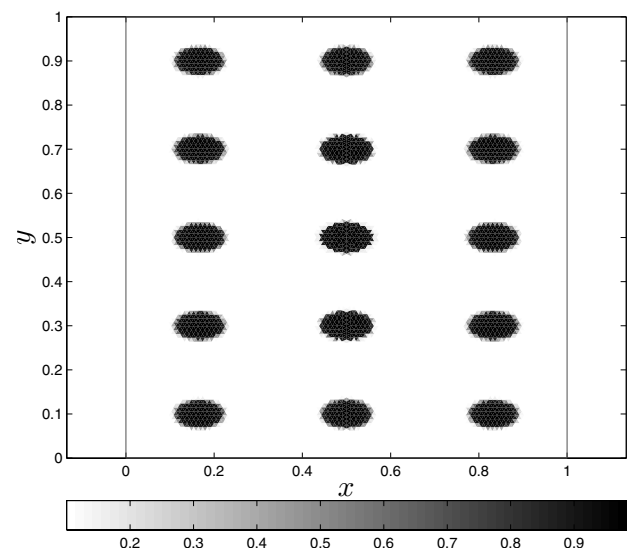

(e) $\alpha=1$

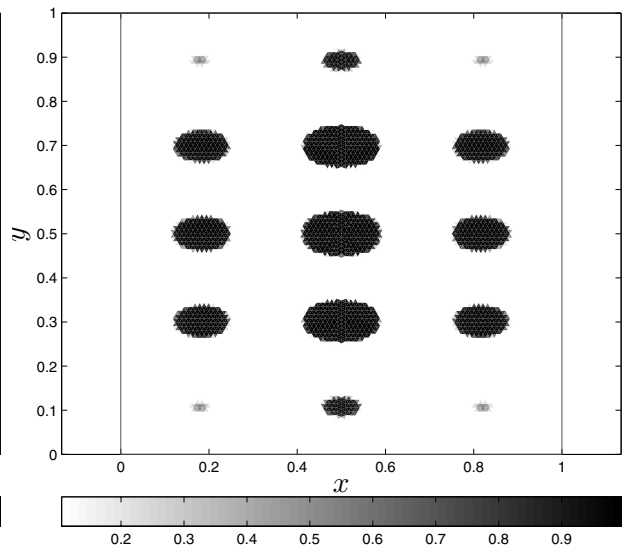

(b) $\alpha=0.7$

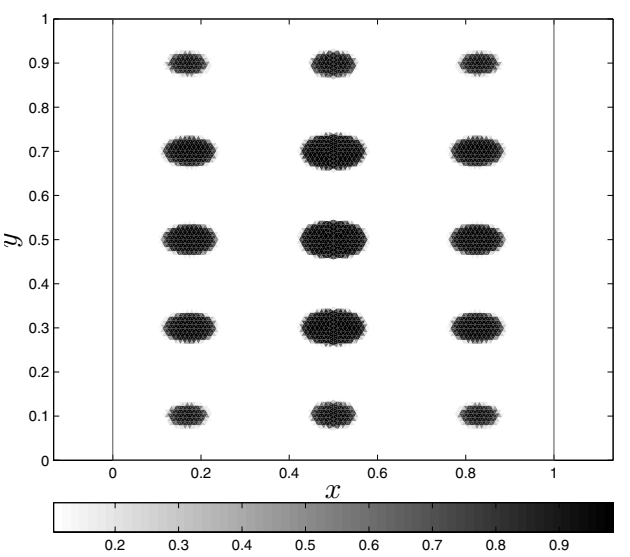

(d) $\alpha=0.9$

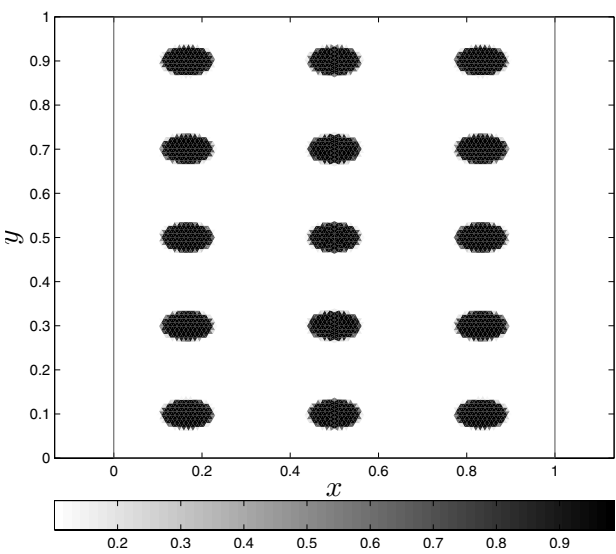

(f) deterministic optimal design

Figure 7. Case 2. Optimal design $s(x, y)$ for different values of $\alpha$. Case (a) corresponds to minimal variance, case (e) to minimal expectation, and case (f) is the optimal deterministic design. 
and the boundary conditions are satisfied a.s. $\omega \in \Omega$. Precisely,

$$
u(\cdot, \omega)=0 \quad \text { on } \Gamma_{D}(\omega) \text { in the sense of traces and a.s. } \omega \in \Omega,
$$

and $\frac{\partial u}{\partial \boldsymbol{n}}(\cdot, \omega)=0$, a.s. $\omega \in \Omega$, as an element of $H^{-1 / 2}\left(\Gamma_{N}(\omega)\right)$, i.e.

$$
\left\langle\frac{\partial u}{\partial \boldsymbol{n}}, \phi\right\rangle=0 \quad \forall \phi \in H^{1 / 2}\left(\Gamma_{N}(\omega)\right)
$$

We notice that, with almost no changes, the theoretical results in Sections 2 and 3 easily extend to the case of problem (5.10). In the numerical implementation of this experiment the level of the $1 \mathrm{D}$ quadrature rule is $k=4$.

Contrary to the previous cases, in this experiment the minimal expectation design differs slightly from the deterministic one. This difference can be explained by the qualitative change in the contours of the solution of problem (5.10) (Fig. 9a) in comparison to the deterministic solution. This change is related to the type of uncertainty introduced into the problem. Figure $9 \mathrm{~b}$ presents the standard deviation of the uncontrolled $(s=0)$ state variable $u(x, \omega)$, showing the maximum deviation at $\theta=0, \pi / 2, \pi, 3 \pi / 2 \mathrm{rad}$.

The parameter for the measure constraint is $L=0.25$. Five single-objective optimization problems are derived from the multi-objective scalarization by modifying the weight factor $\alpha=0,0.25,0.5,0.75,1$. The convergence history of the algorithm is similar to the two previous examples. The Pareto front is depicted in Figure 10. As in the previous cases, the weighted-sum approach allows us effectively approximate the convex Pareto front of optimal solutions. Each solution represents a different configuration of the membrane reinforcement as shown in Figure 8. The most robust design (Fig. 8a) balances the reinforcement between the central region of the domain (Fig. 9a) and the region with a largest deviation of the compliance (Fig. 9b). However, the design with minimal expectation of the compliance concentrates all the allowed reinforcement over a region close to a circle centered at the origin and with radius 0.5 (solution of the deterministic problem).

\section{FURTHER REMARKS AND CONCLUSION}

It is worth pointing out that although the mathematical and numerical approaches presented above have been developed in the context of robust shape optimization, they can be extended to the resolution of other problems in the field of shape optimization under uncertainty such as risk averse shape optimization.

\subsection{Extension to risk averse shape optimization}

The variance is widely used as a measure of deviation in the context of robust optimization $[7,24]$. However, in risk aversion optimization the use of the variance as a risk functional is not the best choice because it treats the excess over the mean equally as the shortfall. This is not appropriate in minimization problems where the main concern is whether the random output is too large. In this section we extend the mathematical and numerical approaches presented in previous sections to be applied to the field of risk averse optimization by using the upper semi-deviation as a risk measure.

Denoting by

$$
X(\omega)=\int_{D} f(x, \omega) u(x, \omega) \mathrm{d} x
$$

the compliance random variable associated to problem (OP), the upper semi-deviation of order 1 of $X$ is defined as $[36]$

$$
E(\max \{X-E(X), 0\})
$$

where $E(X)$ is the expected value of $X$. Since the max-function is not differentiable, it is convenient to approximate it, for instance by using the so-called Chen-Harker-Kanzow-Smale function (see [8]). Thus, a measure of 


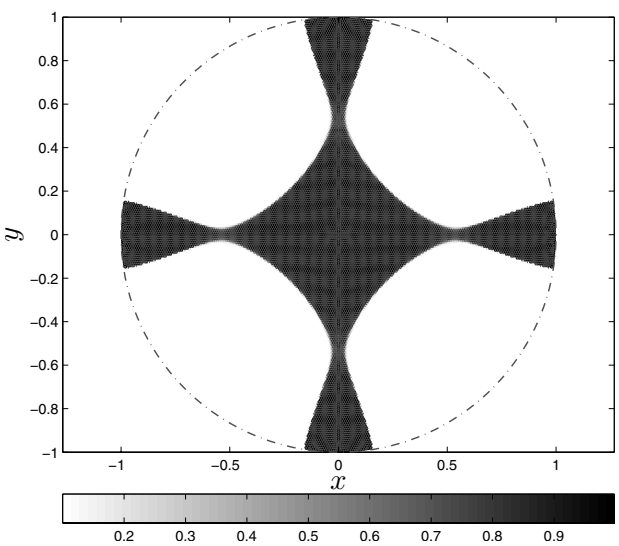

(a) $\alpha=0$

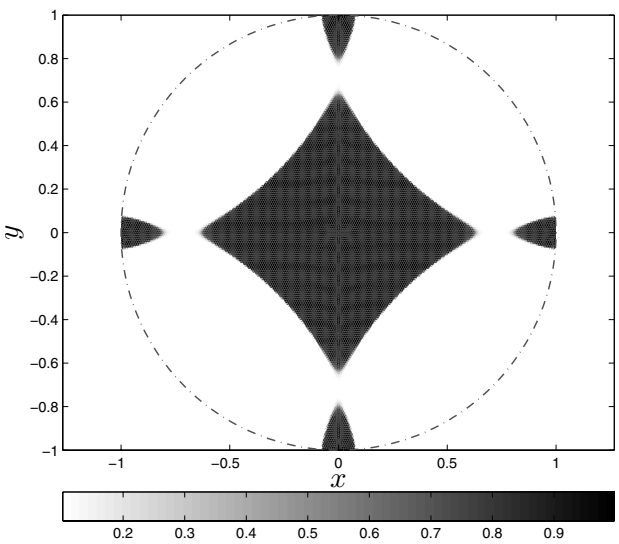

(c) $\alpha=0.5$

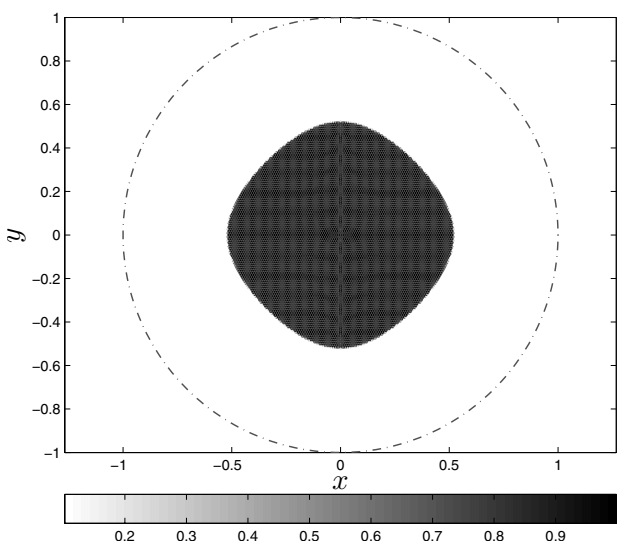

(e) $\alpha=1$

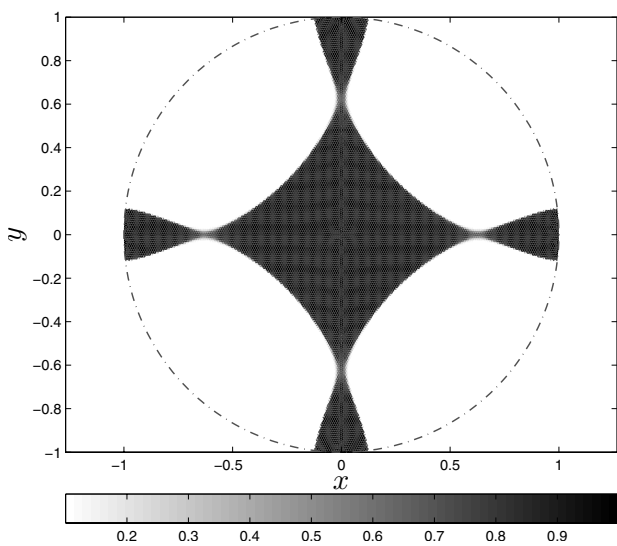

(b) $\alpha=0.25$

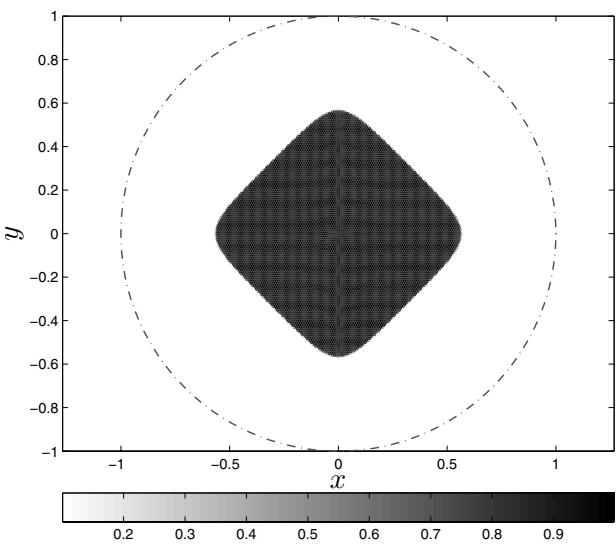

(d) $\alpha=0.75$

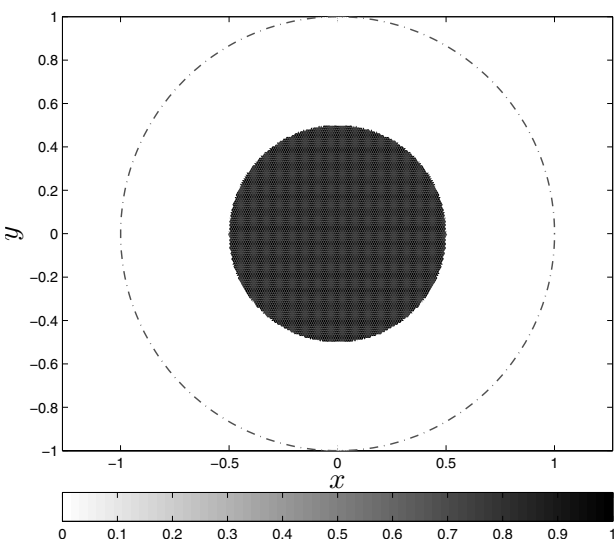

(f) deterministic optimal design

Figure 8. Case 3. Optimal design $s(x, y)$ for different values of $\alpha$. Case (a) corresponds to minimal variance, case (e) to minimal expectation, and case (f) is the optimal deterministic design. 


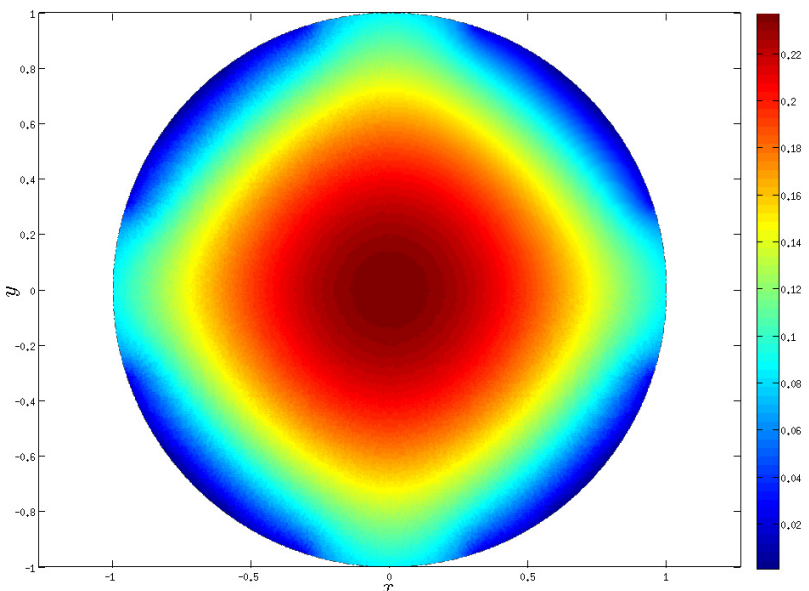

(a)

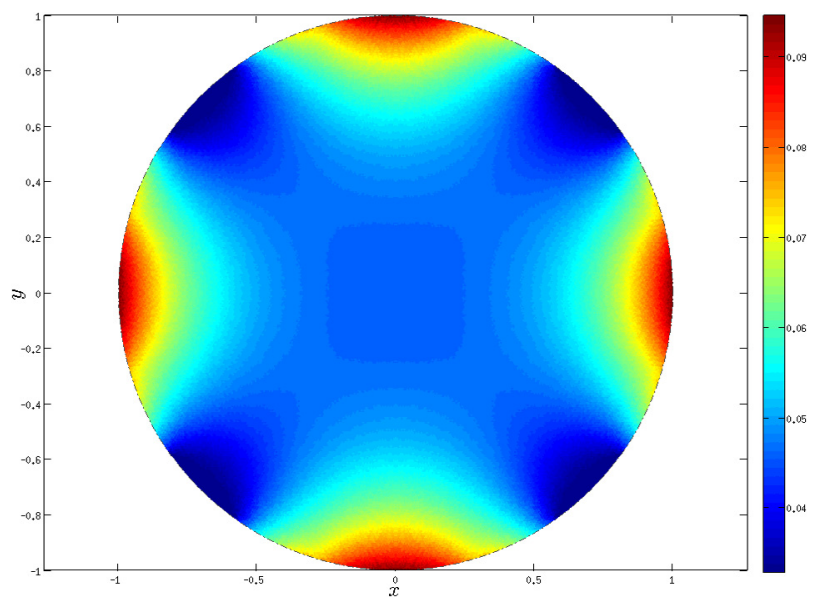

(b)

Figure 9. Case 3: Expectation (a) and standard deviation (b) of the uncontrolled $(s=0)$ solution.

risk aversion is the non-linear functional cost

$$
J_{\varepsilon}\left(1_{\mathcal{O}}\right)=\frac{1}{2} \int_{\Omega}\left[\sqrt{(X(\omega)-E(X))^{2}+\varepsilon}+(X(\omega)-E(X))\right] \mathrm{d} P(\omega),
$$

where $\varepsilon>0$ is a (small enough) parameter.

Similarly to the proof of Theorem 3.1, it can be proved that a relaxed formulation of problem (OP), with the new functional cost (6.2), is obtained by replacing the design variable $1_{\mathcal{O}} \in \mathcal{U}_{L}$ by the density $s \in \overline{\mathcal{U}}_{L}$. Then, the new relaxed cost, from now on denoted by $J_{\varepsilon}(s)$, is Gâteaux differentiable at each admissible $s \in \overline{\mathcal{U}}_{L}$ and its derivative at $s$ is given by

$$
\begin{aligned}
J_{\varepsilon}^{\prime}(s)(x)= & \frac{1}{4} \int_{\Omega} \frac{u_{s}(x, \omega) p_{s}(x, \omega)+2(E(X)) u_{s}^{2}(x, \omega)}{\sqrt{(X(\omega)-E(X))^{2}+\varepsilon}} \mathrm{d} P(\omega) \\
& -\frac{1}{2} \int_{\Omega} u_{s}(x, \omega) q_{s}(x, \omega) \mathrm{d} P(\omega)
\end{aligned}
$$

where $u_{s}$ solves the direct problem (3.2), $p_{s}$ is the solution of (4.2), and $q_{s}$ solves the variational problem: find $q_{s} \in V_{P, a}$ such that

$$
\int_{\Omega} \int_{D}\left[a \nabla q_{s} \cdot \nabla v+s q_{s} v\right] \mathrm{d} x \mathrm{~d} P(\omega)=\int_{\Omega}\left[\int_{D}(f-E(f)) v \mathrm{~d} x\right] \mathrm{d} P(\omega) \quad \forall v \in V_{P, a} .
$$

Once we have computed the gradient (6.3) of the new functional cost, the optimization algorithm proposed in this paper applies to this context.

Using equations (6.2) and (6.3), the numerical experiment presented in Case 3 is extended to the problem of risk averse shape optimization. Only the case of $\alpha=0$ (minimization of upper semi-deviation) is carried out. The optimal solution is compared with the optimal design previously obtained for robust shape optimization (minimization of variance). Both designs are shown in Figure 11. In contrast to the solution obtained minimizing the variance of the compliance, the minimization of the upper semi-deviation leads to designs in which the reinforcement is totally placed over a region close to the lost boundary constraint. The results obtained highlight the differences between both approaches: whereas in risk averse optimization particular care is paid on minimizing the risk of the system in extreme events, in the robust design optimization more emphasis is put on the system performance under everyday fluctuations. 


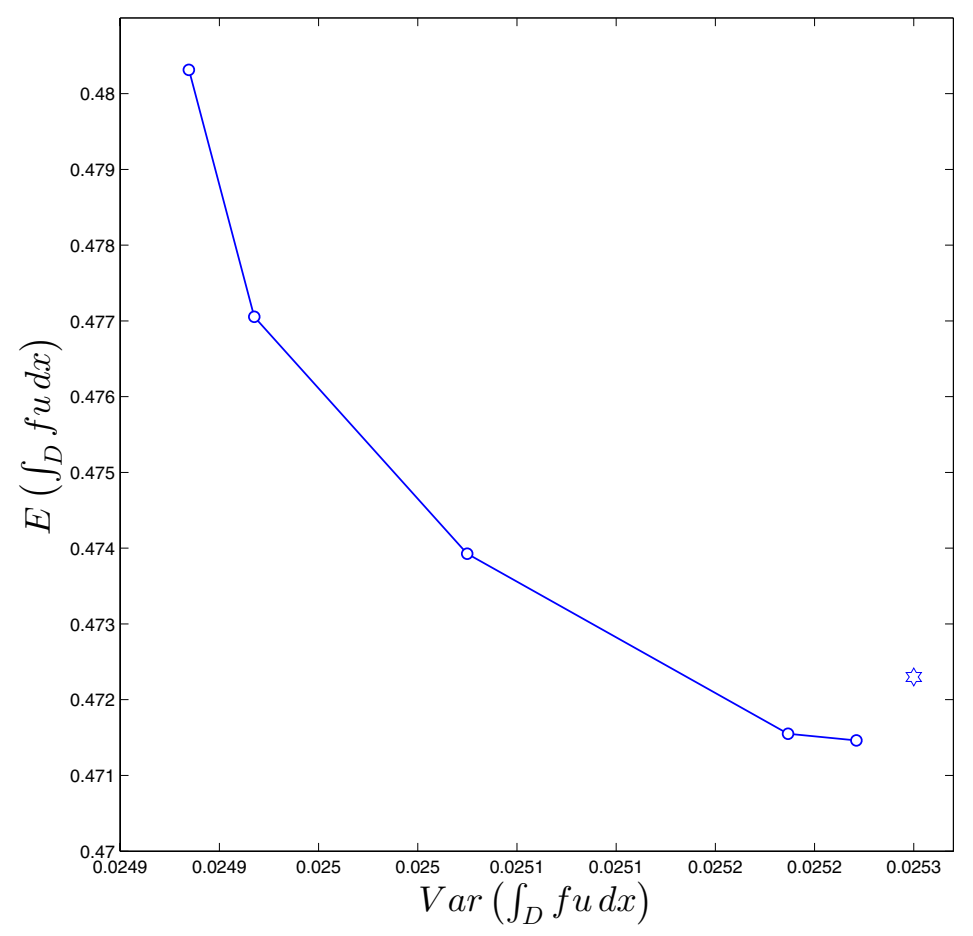

Figure 10. Case 3. Pareto front of optimal solutions (circles) and deterministic solution (star). From left to right, and top to bottom, the circles correspond to the values $\alpha=0,0.25,0.5,0.75$ and 1.

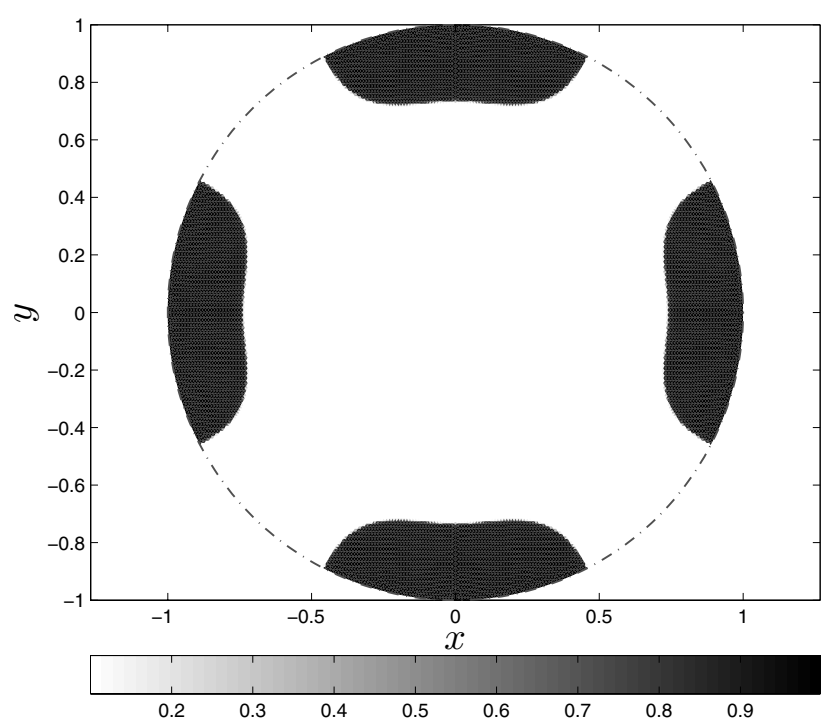

(a)

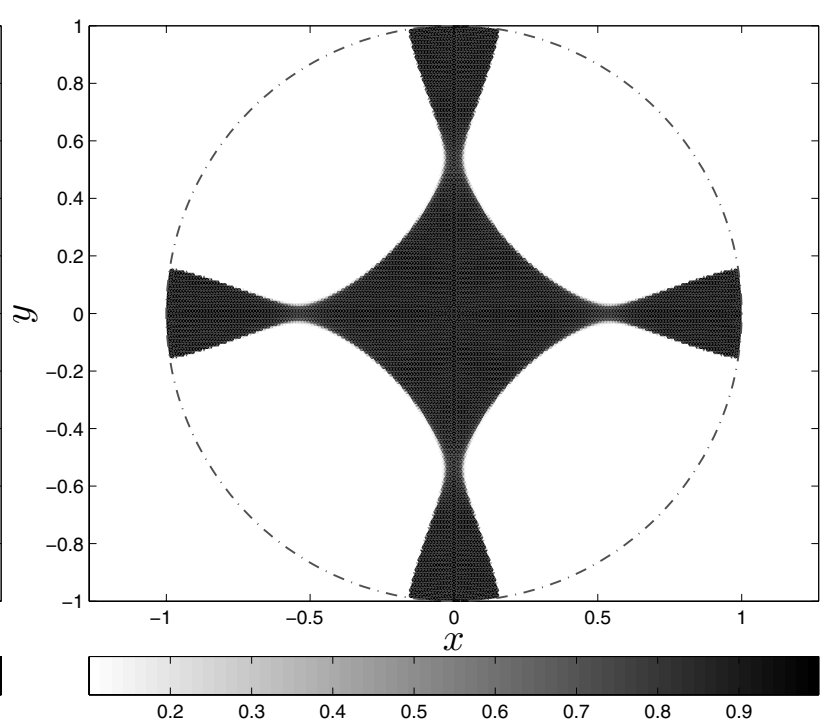

(b)

Figure 11. Case 3. Optimal design $s(x, y)$ for $\alpha=0$. Case (a) corresponds to risk averse shape optimization, case (b) to robust shape optimization. 
We notice that the approach described in this paper may be adapted to deal with other risk aversion measures like semi-deviations below some specific target, weighted mean deviations from quantiles or from a preselected tolerance level, etc. (we refer the reader to $[19,27,36]$ for more details).

\subsection{Conclusion}

In this paper, a robust shape optimization problem for an elliptic PDE under uncertainties in the diffusion coefficient, force term and boundary conditions has been addressed. Previous works in this field [14, 25, 37] address the robust shape optimization problem through semi-analytical expressions of the gradient of the cost functional, which are obtained from a discrete version of the state equation both in the physical and in the stochastic domains. This paper proposes a continuous approach for robust shape optimization. Since the space of admissible shapes is not convex, a well posed full relaxation of the original problem is obtained. The relaxed problem is numerically solved by using a descent method which is based on the computation of the continuous gradient of the cost function. To this end, the adjoint method is used leading to the numerical resolution of both the direct and the adjoint equations. The main contributions of the present paper are within the field of robust shape optimization. Indeed, up to the best knowledge of the authors, this continuous approach (considering the variance in the cost function and uncertainties modeled by Gaussian fields entering in all the input data of the problem, namely the diffusion coefficient, force term and boundary conditions) has not been addressed so far. Finally, it is illustrated that the approach proposed in this paper can be extended to other formulations of the problem of shape optimization under uncertainty such us risk averse shape optimization.

Acknowledgements. Acknowledgements. We acknowledge the use of the Matlab package spinterp developed by Dr. Andreas Klimke from Universität Stuttgart http://www.ians.uni-stuttgart.de/spinterp/ and the Matlab code of the MMA algorithm provided by Krister Svanberg. The third author was partially supported by projects MTM201019739 from "Ministerio de Educación y Ciencia (Spain)" and 08720/PI/08 from "Fundación Séneca (Agencia de Ciencia y Tecnología de la Región de Murcia (Spain)" under contract II PCTRM 2007-10).

\section{REFERENCES}

[1] G. Allaire, Shape Optimization by the Homogenization Method. Vol. 146 of Appl. Math. Sci. Springer-Verlag, New York (2002).

[2] M. Allen and K. Maute, Reliability-based shape optimization of structures undergoing fluid-structure interaction phenomena. Comput. Methods Appl. Mech. Engrg. 194 (2005) 3472-3495.

[3] F. Alvarez and M. Carrasco, Minimization of the expected compliance as an alternative approach to multiload truss optimization. Struct. Multidisc. Optim. 29 (2005) 470-476.

[4] A. Asadpoure, M. Tootkaboni and J.K. Guest, Robust topology optimization of structures with uncertainties in stiffness: application to truss structures. Comput. Struct. 89 (2011) 1131-1141.

[5] I. Babuška, F. Novile and R. Tempone, A Stochastic collocation method for elliptic partial differential equations with random input data. SIAM Rev. 52 (2010) 317-355.

[6] M.P. Bensøe and O. Sigmund, Topology Optimization: Theory, Methods and Applications. Springer-Verlag, Berlin (2003).

[7] H.-G. Beyer and B. Sendhoff, Robust optimization â a comprehensive survey. Comput. Methods Appl. Mech. Engrg. 196 (2007) 3190-3218.

[8] S.I. Birbil, S.C. Fang, J.B.G. Frenk and S. Zhang, Recursive approximation of the high dimensional max function. Oper. Res. Lett. 33 (2005) 450-458.

[9] D. Buccur and G. Buttazzo, Variational Methods in Shape Optimization Problems. Progr. Non Lin. Differ. Eq. Appl. Birkhäuser Boston, Inc., Boston, MA (2005).

[10] G. Buttazzo, N. Varchon and H. Zoubairi, Optimal measures for elliptic problems. Ann. Mat. Pura Appl. 185 (2006) $207-221$.

[11] G. Buttazzo and F. Maestre, Optimal shape for elliptic problems with random perturbations. Discrete Contin. Dyn. Syst. 31 (2011) 1115-1128.

[12] G.C. Calafiore and F. Dabbene, Optimization under uncertainty with applications to design of truss structures. Struct. Multidisc. Optim. 35 (2008) 189-200.

[13] J. Casado-Díaz, C. Castro, M. Luna-Laynez and E. Zuazua, Numerical approximation of a one-dimensional elliptic optimal design problem. Multiscale Model. Simul. 9 (2011) 1181-1216.

[14] S. Chen, W. Chen and S. Lee, Level set based robust shape and topology optimization under random field uncertainties. Struct. Multidisc. Optim. 41 (2010) 507-524.

[15] A. Cherkaev, Variational Methods for Structural Optimization. Vol. 140 of Appl. Math. Sci. Springer-Verlag, New York (2000).

[16] A. Cherkaev and E. Cherkaeva, Principal compliance and robust optimal design. J. Elasticity 72 (2003) 71-98. 
[17] J. Cohon, Multiobjective Programming and Planning. Dover Publications (2004).

[18] S. Conti, H. Held, M. Pach, M. Rumpf and R. Schultz, Shape optimization under uncertainty - A stochastic programming approach. SIAM J. Optim. 19 (2009) 1610-1632.

[19] S. Conti, H. Held, M. Pach, M. Rumpf and R. Schultz, Risk Averse Shape Optimization. SIAM J. Control Optim. 49 (2011) 927-947.

[20] M. Davis, Production of Conditional Simulations via the LU Triangular Decomposition of the Covariance Matrix. J. Math. Geol. 19 (1987) 91-98.

[21] F. de Gournay, G. Allaire and F. Jouve, Shape and topology optimization of the robust compliance via the level set method. ESAIM:COCV 14 (2008) 43-70.

[22] X. Guo, W. Zhang and L. Zhang, Robust structural topology optimization considering boundary uncertainties. Comput. Methods Appl. Mech. Engrg. 253 (2013) 356-368.

[23] E. de Rocquigny, N. Devictor, S. Tarantola, Uncertainty in Industrial Practice: A Guide to Quantitative Uncertainty Management. John Wiley (2008).

[24] I. Doltsinis and Z. Kang, Robust design of structures using optimization methods. Comput. Methods Appl. Mech. Engrg. 193 (2004) 2221-2237.

[25] P.D. Dunning and H.A. Kim, Robust Topology Optimization: Minimization of Expected and Variance of Compliance. AIAA J. 51 (2013) 2656-2664.

[26] I. Enevoldsen and J.D. Sørensen, Reliability-based optimization in structural engineering. Struct. Safety 15 (1994) $169-196$.

[27] B. Geihe, M. Lenz, M. Rumpf and R. Schultz, Risk averse elastic shape optimization with parametrized fine scale geometry. Math. Program. 141 (2013) 1-2.

[28] W. Hager, Runge-Kutta methods in optimal control and the transformed adjoint system. Numer. Math. 87 (2000) $247-282$.

[29] A. Henrot and H. Maillot, Optimization of the shape and the location of the actuators in an internal control problem. Bolletino U.M.I. 8 (2001) 737-757.

[30] A. Henrot and M. Pierre, Variation et optimization de formes. Math. Appl. Springer (2005).

[31] D.W. Kim and B.M. Kwak, Reliability-based shape optimization of two-dimensional elastic problems using BEM. Comput. Struct. 60 (1996) 743-750.

[32] M. Loève, Probability Theory. I, 4th edition. Vol. 45 of Grad. Texts Math. Springer-Verlag, New York (1977).

[33] M. Loève, Probability Theory. II, 4th edition. Vol. 46 of Grad. Texts Math. Springer-Verlag, New York (1978).

[34] R.T. Marler and J.S. Arora, Survey of multi-objective optimization methods for engineering. Struct. Multidisc. Optim. 26 (2004) 369-395.

[35] G.I. Schuëller and H.A. Jensen, Computational methods in optimization considering uncertainties - An overview. Comput. Methods Appl. Mech. Engrg. 198 (2008) 2-13.

[36] A. Shapiro, D. Dentcheva and A. Ruszczynski, Lectures on Stochastic Programming. Modelling and Theory. MPS-SIAM Ser. Optim. (2009).

[37] C. Shikui and C. Wei, A new level-set based approach to shape and topology optimization under geometric uncertainty. Struct. Multidisc. Optim. $44(2011) 1-18$.

[38] S.A. Smolyak, Quadrature and interpolation formulas for tensor products of certain classes of functions. Doklady Akademii Nauk SSSR 4 (1963) 240-243.

[39] J. Sokolowski and J.P. Zolésio, Introduction to Shape Optimization: Shape Sensitivity Analysis. Vol. 16 of Springer Series Comput. Math. Springer-Verlag, Berlin (1992).

[40] K. Svanberg, The method of moving asymptotes - a new method for structural optimization. Int. J. Numer. Methods Engrg. 24 (1987) 359-373.

[41] K. Svanberg, A class of globally convergent optimization methods based on conservative convex separable approximations. SIAM J. Optim. 12 (2002) 555-573.

[42] N. Varchon, Optimal measures for nonlinear cost functionals. Appl. Math. Optim. 54 (2006) 205-221.

[43] N. Wiener, The homogeneous chaos. Amer. J. Math. 60 (1938) 897-936.

[44] E. Zuazua, Propagation, Observation, Control and Numerical Approximation of Waves approximated by finite difference method. SIAM Rev. 47 (2005) 197-243. 